\title{
TECHNISCHE UNIVERSITÄT CHEMNITZ
}

\author{
Michael Weise
}

A framework for efficient hierarchic plate and shell elements

$\mathrm{CSC} / 17-01$

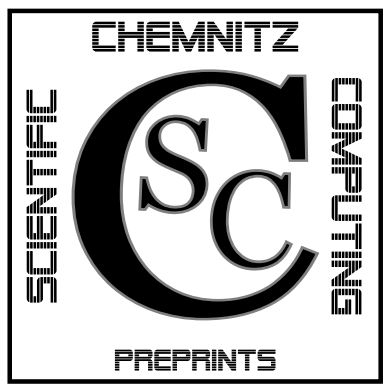

Chemnitz Scientific Computing

Preprints 


\section{Impressum:}

Chemnitz Scientific Computing Preprints - ISSN 1864-0087 (1995-2005: Preprintreihe des Chemnitzer SFB393)

Herausgeber:

Professuren für

Numerische und Angewandte Mathematik an der Fakultät für Mathematik der Technischen Universität Chemnitz
Postanschrift:

TU Chemnitz, Fakultät für Mathematik 09107 Chemnitz

Sitz:

Reichenhainer Str. 41, 09126 Chemnitz

http://www.tu-chemnitz.de/mathematik/csc/ 


\title{
TECHNISCHE UNIVERSITÄT CHEMNITZ \\ Chemnitz Scientific Computing \\ Preprints
}

\author{
Michael Weise
}

\section{A framework for efficient hierarchic plate and shell elements}

\author{
$\mathrm{CSC} / 17-01$
}

\begin{abstract}
The Mindlin-Reissner plate model is widely used for the elastic deformation simulation of moderately thick plates. Shear locking occurs in the case of thin plates, which means slow convergence with respect to the mesh size. The Kirchhoff plate model does not show locking effects, but is valid only for thin plates. One would like to have a method suitable for both thick and thin plates.

Several approaches are known to deal with the shear locking in the Mindlin-Reissner plate model. In addition to the well known MITC elements and other approaches based on a mixed formulation, hierarchic methods have been developed in the recent years. These are based on the Kirchhoff model and add terms to account for shear deformations.

We present some of these methods and develop a new hierarchic plate formulation. This new model can be discretised by a combination of $C^{0}$ and $C^{1}$ finite elements. Numerical tests show that the new formulation is locking free and numerically efficient.

We also give an extension of the model to a hierarchic Naghdi shell based on a Koiter shell formulation with unknowns in Cartesian coordinates.
\end{abstract}




\section{Contents}

1 Introduction 1

2 Plate theory 2

2.1 A simple benchmark problem . . . . . . . . . . . . . . . 2

2.2 Basic assumptions and formulas . . . . . . . . . . . . . . . . . 3

2.3 The standard Mindlin-Reissner plate formulation (MRs) . . . . . . . . . 4

2.4 The Kirchhoff plate . . . . . . . . . . . . . . . . . . . 5

2.5 The Mindlin-Reissner plate in hierarchic formulation (MRh) . . . . . . 5

2.6 The Mindlin-Reissner plate in a rotation free formulation by Oesterle, Ramm and Bischoff $(\mathrm{ORB}) \ldots \ldots \ldots$. . . . . . . . . . 7

2.7 The plate formulation of Endo and Kimura $(\mathrm{EKs}) \ldots \ldots \ldots$

2.8 The Endo-Kimura plate in hierarchic formulation $(\mathrm{EKh}) \ldots \ldots$

2.9 First new formulation: Endo-Kimura plate decoupled (EKd) . . . . . . . 9

2.10 Second new formulation: hierarchic Mindlin-Reissner based on EndoKimura $(\mathrm{MREK}) \ldots \ldots \ldots$. . . . . . . . . . . . . . 10

2.11 Comparison of numerical results . . . . . . . . . . . . . . . . 11

3 Shell theory 16

3.1 The Naghdi shell . . . . . . . . . . . . . . . . . . . . . . . 16

3.1.1 Naghdi shell in covariant coordinates . . . . . . . . . . . . . . . . 16

3.1.2 Coordinate free Naghdi shell formulation . . . . . . . . . . . . . . 17

3.2 The Koiter shell . . . . . . . . . . . . . . . . . . . . . . . . 18

3.2.1 Coordinate free Koiter shell formulation . . . . . . . . . . . . 18

3.2 .2 Koiter shell in Cartesian coordinates . . . . . . . . . . . . . . . . 19

3.3 The principle of Endo-Kimura applied to shells . . . . . . . . . . . . . 19

3.4 Hierarchic Naghdi shell formulation based on Endo-Kimura . . . . . . . 20

3.5 Numerical example: Scordelis-Lo roof . . . . . . . . . . . . . . . . . 21

4 Conclusion

Author's address:

Michael Weise

TU Chemnitz, Fakultät für Mathematik

09107 Chemnitz, Germany

http://www.tu-chemnitz.de/mathematik/ 


\section{Introduction}

The Mindlin-Reissner plate model is widely used for the elastic deformation simulation of moderately thick plates. The weak formulation of the model features functions from $H^{1}$ and can thus be discretised with $C_{0}$ finite elements. This simple approach works if the considered plate is rather thick, but it leads to problems when used with thin plates. One observes very slow convergence with respect to the mesh size. Meshes sufficiently fine for a thick plate may yield results which are several orders of magnitude too small in case of a thin plate. This effect is called shear locking in the engineering literature due to the fact that the plate seems to be stiffer than it is with an insufficently fine mesh.

On the other hand, the Kirchhoff plate model does not show such locking effects, but is valid only for thin plates. It excludes out-of-plane shear deformations, which are negligible for thin plates but relevant for thick plates. The weak formulation features functions from $H^{2}$ and therefore requires $C_{1}$ finite elements for a conforming discretisation.

One would like to have a method suitable for both thick and thin plates. Several approaches are known to deal with the shear locking in the Mindlin-Reissner model. In addition to the well known MITC elements and other approaches based on a mixed formulation, hierarchic methods have been developed in the recent years. We focus on hierarchic methods in this article and present two new formulations. In fact we will not really discuss specific elements, but different formulations of the plate theory which may then be discretised with suitable elements.

We will present and discuss numerical examples achieved with two combinations of $C_{0}$ and $C_{1}$ elements: linear Lagrange ansatz functions and reduced Hsieh-Clough-Tocher (rHCT) ansatz functions for triangular elements and bilinear Lagrange ansatz functions and Bogner-Fox-Schmit (BFS) ansatz functions for rectangular elements. See for example [7], [1], [17] for details on rHCT elements and [3], [13] for details on BFS elements.

The article is structured as follows. Section 2 deals with plate formulations. A simple benchmark problem is given in 2.1. Subsection 2.2 introduces some basic concepts and formulas for the plate problem. Subsections 2.3 to 2.10 present known and new plate formulations and give a short assessment of their performance with conforming elements based on the benchmark problem from 2.1. A more thorough numerical comparison is presented in subsection 2.11. Section 3 presents the basic concepts of the Naghdi and Koiter shell theories in 3.1 and 3.2 and extends the new plate formulations to shells in 3.3 and 3.4 with numerical examples in 3.5. The article is concluded in section 4. 


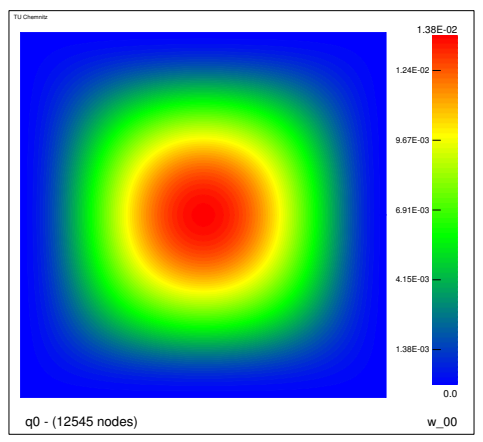

Figure 1: Plate deflection under load

\section{Plate theory}

\subsection{A simple benchmark problem}

Before presenting the different plate formulations we define a benchmark problem which will be used for a first assessment of each theory in the following sections. A deeper analysis and comparison of the numerical performance is then given in section 2.11.

We consider a square plate of length 1 with isotropic material, i. e. same material behaviour in all space directions. All edges are hard clamped which means no deflection and no bending angle. This reads $w=\theta=0$ in the variables defined in the following section. A thick plate with thickness $d=10^{-1}$ and a thin plate with thickness $d=10^{-3}$ are subjected to a scaled load of $d^{3}$. See Figure 1 for the qualitative resulting deflection in the thin plate case. Rough reference solutions for the maximum deflection are $1.60 \times 10^{-2}$ for the thick plate and $1.38 \times 10^{-2}$ for the thin plate.

The first assessment is done with uniform refinements of one quadratic finite element comprising the whole plate as initial coarse mesh. The full comparison in section 2.11 also features triangular elements. $C_{0}$ linear Lagrange ansatz functions and $C_{1}$ reduced Hsieh-Clough-Tocher ansatz functions are used for triangular elements and $C_{0}$ bilinear Lagrange ansatz functions and $C_{1}$ Bogner-Fox-Schmit ansatz functions are used for quadratic elements.

The FE system is solved with the preconditioned conjugate gradient method. Hierarchic preconditioning is used, see [11]. The relative decrease of the residual in the preconditioned norm

$$
\left(r_{k}^{\top} w_{k}\right)^{1 / 2}<\operatorname{tol}\left(r_{0}^{\top} w_{0}\right)^{1 / 2}
$$

with residuals and preconditioned residuals $r_{k}, w_{k}$ of iteration $k$ and $t o l=10^{-4}$ serves as stopping criterion. The initial residual $r_{0}$ is computed from a zero solution on the initial 


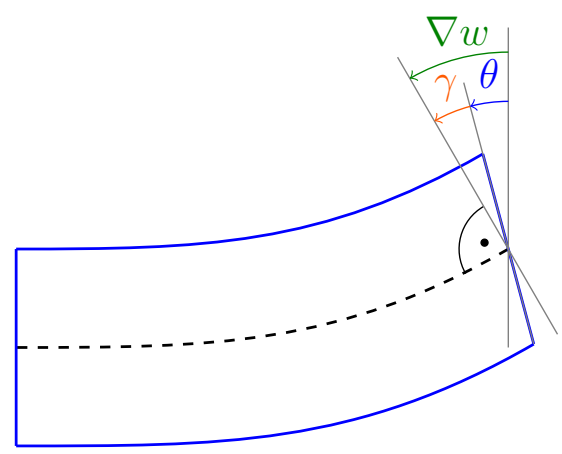

Figure 2: Deformed plate from one side; thickness exaggerated

coarse mesh and from an interpolated solution of the next coarser mesh on all refined meshes.

\subsection{Basic assumptions and formulas}

Figure 2 depicts a deformed plate with extremely exaggerated thickness viewed from the side. The Mindlin-Reissner hypothesis states that a straight line vertical to the plate midsurfaces remains a straight line after deformation, possibly with a different angle than before. The angle between the original line and the same line after deformation is called the bending angle $\theta$. We abbreviate the spatial derivative $\partial \bullet / \partial x_{i}$ of any object $\bullet$ with an index $\bullet_{i}$ throughout this section. The $2 \mathrm{D}$ gradient of the vertical deflection $w$, $\nabla w=\left[w_{, 1}, w_{, 2}\right]^{\top}=\left[\partial w / \partial x_{1}, \partial w / \partial x_{2}\right]^{\top}$, can also be viewed as an angle. This allows for the definition of the shear angle $\gamma$ by $\gamma=\nabla w-\theta$ or, equivalently,

$$
\nabla w=\theta+\gamma .
$$

The 3D deformation of a point in the plate domain $\left(\eta^{1}, \eta^{2}, \tau\right) \in \omega \times[-d / 2, d / 2], \omega \subset \mathbb{R}^{2}$ under the Mindlin-Reissner hypothesis may then be described by

$$
u^{3 \mathrm{D}}\left(x_{1}, x_{2}, x_{3}\right)=\left[\begin{array}{c}
-x_{3} \theta_{1}\left(x_{1}, x_{2}\right) \\
-x_{3} \theta_{2}\left(x_{1}, x_{2}\right) \\
w\left(x_{1}, x_{2}\right)
\end{array}\right] .
$$

We collect the unknowns for any of the following plate formulations in a vector called $u$. Plugging the deformation ansatz (2) into the 3D elasticity bilinear form and integrating over the thickness direction variable $x_{3}$ results in the problem

$$
\text { find } u \in \mathbb{V} \text { with } a(u, \widetilde{u})=l(\widetilde{u}) \quad \forall \widetilde{u} \in \mathbb{V}_{\text {test }}
$$

with the bilinear form

$$
a(u, \widetilde{u})=\int_{\omega} \varepsilon(\theta): \mathfrak{C}^{\mathrm{b}}: \varepsilon(\widetilde{\theta}) \mathrm{d} \omega+\int_{\omega} \gamma \cdot \mathcal{C}^{\mathrm{s}} \cdot \widetilde{\gamma} \mathrm{d} \omega,
$$




\begin{tabular}{r|rrrr|rrrr}
\hline & \multicolumn{2}{|c|}{ bilin., thick plate } & \multicolumn{2}{c}{ bilin., thin plate } & \multicolumn{2}{c}{ biquadr., thick plate } & \multicolumn{2}{c}{ biquadr., thin plate } \\
\#E1 & $w_{\max }$ & $\#$ \#t & $w_{\max }$ & \#it & $w_{\max }$ & \#it & $w_{\max }$ & \#it \\
\hline 4 & $2.44 \mathrm{E}-3$ & 2 & $2.44 \mathrm{E}-7$ & 2 & $8.82 \mathrm{E}-3$ & 4 & $1.39 \mathrm{E}-6$ & 4 \\
16 & $6.67 \mathrm{E}-3$ & 9 & $9.26 \mathrm{E}-7$ & 9 & $1.49 \mathrm{E}-2$ & 24 & $4.95 \mathrm{E}-6$ & 42 \\
64 & $1.17 \mathrm{E}-2$ & 24 & $3.43 \mathrm{E}-6$ & 38 & $1.59 \mathrm{E}-2$ & 42 & $4.88 \mathrm{E}-3$ & 441 \\
256 & $1.46 \mathrm{E}-2$ & 40 & $1.34 \mathrm{E}-5$ & 114 & $1.60 \mathrm{E}-2$ & 42 & $1.08 \mathrm{E}-2$ & 1444 \\
1024 & $1.56 \mathrm{E}-2$ & 48 & $5.31 \mathrm{E}-5$ & 286 & $1.60 \mathrm{E}-2$ & 35 & $1.23 \mathrm{E}-2$ & 227 \\
4096 & $1.59 \mathrm{E}-2$ & 49 & $2.10 \mathrm{E}-4$ & 662 & $1.60 \mathrm{E}-2$ & 33 & $1.29 \mathrm{E}-2$ & 891 \\
16384 & $1.60 \mathrm{E}-2$ & 47 & $8.01 \mathrm{E}-4$ & 1489 & $1.60 \mathrm{E}-2$ & 32 & $1.32 \mathrm{E}-2$ & 1426 \\
\hline
\end{tabular}

Table 1: Results for the standard Mindlin-Reissner plate formulation with $C^{0}$ elements

the linear form

$$
l(\widetilde{u})=\int_{\omega} p \widetilde{w} \mathrm{~d} \omega
$$

and appropriate $\mathrm{FE}$ ansatz and test spaces $\mathbb{V}$ and $\mathbb{V}_{\text {test }}$. The linearised 2D strain tensor $\varepsilon(\bullet)=\frac{1}{2}\left(\nabla \bullet+(\nabla \bullet)^{\top}\right)$ is given as the symmetrised $2 \mathrm{D}$ gradient. The fourth order material bending tensor $\mathfrak{C}^{\mathrm{b}}$ and the second order shear tensor $\mathcal{C}^{\mathrm{s}}$ are proportional to $d^{3}$ and $d$, respectively, due to the thickness integration.

The given formulation of the problem and the bilinear and linear forms features the three variables $w, \theta, \gamma$. The relation $\nabla w=\theta+\gamma$ from (1) allows the elimination of either $\theta$ or $\gamma$ from the problem. This leads to differing formulations with different numerical behaviour which are explored in the following sections.

\subsection{The standard Mindlin-Reissner plate formulation (MRs)}

The standard formulation of the Mindlin-Reissner plate problem follows from eliminating $\gamma=\nabla w-\theta$ from the three variable formulation (2)-(5). This leads to the bilinear form

$$
a(u, \widetilde{u})=\int_{\omega} \varepsilon(\theta): \mathfrak{C}^{\mathrm{b}}: \varepsilon(\widetilde{\theta}) \mathrm{d} \omega+\int_{\omega}(\nabla w-\theta) \cdot \mathcal{C}^{\mathrm{s}} \cdot(\nabla \widetilde{w}-\widetilde{\theta}) \mathrm{d} \omega
$$

with the unknowns $u=\left[w, \theta_{1}, \theta_{2}\right]^{\top}$. All unknowns are featured with derivatives up to first order and are thus assumed to be in $H^{1}(\omega)$. This allows for a simple discretisation with $C^{0}$ finite elements.

Results for the example problem from section 2.1 with bilinear and biquadratic rectangular elements are shown in Table 1 . One observes reasonable $h$-convergence for the thick plate case but very slow convergence for the thin plate case. This effect is called thickness locking or shear locking. Standard finite elements are not capable of resolving the first (bending) term and the second (shear) term of the bilinear form appropriately for small thickness $d$. As $d$ tends to zero, the shear term dominates due to the scaling of the bending term with $d^{3}$ and the shear term with $d$. The shear term is imbalanced in the sense that it consists of a function value and derivative value. 


\begin{tabular}{rrrrr}
\hline & \multicolumn{2}{c}{ thick plate } & \multicolumn{2}{c}{ thin plate } \\
\#E1 & $w_{\max }$ & \#it & $w_{\max }$ & \#it \\
\hline 4 & $1.45 \mathrm{E}-2$ & 2 & $1.45 \mathrm{E}-2$ & 2 \\
16 & $1.38 \mathrm{E}-2$ & 8 & $1.38 \mathrm{E}-2$ & 8 \\
64 & $1.38 \mathrm{E}-2$ & 23 & $1.38 \mathrm{E}-2$ & 22 \\
256 & $1.38 \mathrm{E}-2$ & 35 & $1.38 \mathrm{E}-2$ & 35 \\
1024 & $1.38 \mathrm{E}-2$ & 29 & $1.38 \mathrm{E}-2$ & 29 \\
4096 & $1.38 \mathrm{E}-2$ & 29 & $1.38 \mathrm{E}-2$ & 28 \\
16384 & $1.38 \mathrm{E}-2$ & 31 & $1.38 \mathrm{E}-2$ & 83 \\
\hline
\end{tabular}

Table 2: Results for the Kirchhoff plate problem with $C^{1}$ BFS elements

\subsection{The Kirchhoff plate}

The plate model of Kirchhoff can be viewed as a special case of the Mindlin-Reissner plate without the allowance for a shear angle. The condition $\gamma=0$ gives $\theta=\nabla w$ from (1). The geometric interpretation of this ansatz is that orthogonal line elements prior to deformation are still orthogonal to the new plate midsurface after deformation. The problem formulation changes to

$$
u^{3 \mathrm{D}}=\left[\begin{array}{c}
-x_{3} w_{, 1} \\
-x_{3} w_{, 2} \\
w
\end{array}\right], \quad a(u, \widetilde{u})=\int_{\omega} \varepsilon(\nabla w): \mathfrak{C}^{\mathrm{b}}: \varepsilon(\nabla \widetilde{w}) \mathrm{d} \omega, \quad l(\widetilde{u})=\int_{\omega} p \widetilde{w} \mathrm{~d} \omega
$$

with the single unknown $u=w$. The bilinear form features second derivatives which calls for $w \in H^{2}(\omega)$. Thus, $C^{1}$ finite elements are needed for a conforming discretisation.

Results for the example problem from section 2.1 with BFS elements are shown in Table 2. Even very coarse meshes yield a quite accurate solution for the thin plate. No shear locking is observed because no shear term is present. The thick plate example converges equally fast, but to the same value of $1.38 \times 10^{-2}$ while the reference solution for the thick plate is about $1.60 \times 10^{-2}$. This is due to the fact that the Kirchhoff model neglects the shear term and is thus only suitable for thin plates.

\subsection{The Mindlin-Reissner plate in hierarchic formulation (MRh)}

The desire for a suitable plate formulation for thick and thin plates has lead to numerous reformulations of the Mindlin-Reissner plate problem with the goal of the exclusion of locking. A hierarchic approach can be found in [8] based on earlier works, see the references therein. The cited work uses isogeometric analysis (finite elements with nonuniform rational B-splines used for geometry definition and as basis functions) but the formulation given there can also be discretised with standard finite elements. 


\begin{tabular}{rrrrr}
\hline & \multicolumn{2}{c}{ thick plate } & \multicolumn{2}{c}{ thin plate } \\
\#E1 & $w_{\max }$ & \#it & $w_{\max }$ & \#it \\
\hline 4 & $1.45 \mathrm{E}-2$ & 2 & $1.45 \mathrm{E}-2$ & 2 \\
16 & $1.53 \mathrm{E}-2$ & 10 & $1.38 \mathrm{E}-2$ & 9 \\
64 & $1.56 \mathrm{E}-2$ & 32 & $1.38 \mathrm{E}-2$ & 24 \\
256 & $1.58 \mathrm{E}-2$ & 73 & $1.38 \mathrm{E}-2$ & 45 \\
1024 & $1.59 \mathrm{E}-2$ & 130 & $1.38 \mathrm{E}-2$ & 68 \\
4096 & $1.60 \mathrm{E}-2$ & 214 & $1.38 \mathrm{E}-2$ & 92 \\
16384 & $1.60 \mathrm{E}-2$ & 423 & $1.38 \mathrm{E}-2$ & 108 \\
\hline
\end{tabular}

Table 3: Results for the hierarchic Mindlin-Reissner formulation with $C^{1}$ BFS elements for $w$ and $C^{0}$ bilinear elements for $\gamma$

The idea is to eliminate $\theta=\nabla w-\gamma$ from the three variable formulation (2)-(5) instead of $\gamma$. This leads to

$$
\begin{aligned}
u^{3 \mathrm{D}} & =\left[\begin{array}{c}
-x_{3}\left(w_{, 1}-\gamma_{1}\right) \\
-x_{3}\left(w_{, 2}-\gamma_{2}\right) \\
w
\end{array}\right], \\
a(u, \widetilde{u}) & =\int_{\omega} \varepsilon(\nabla w-\gamma): \mathfrak{C}^{\mathrm{b}}: \varepsilon(\nabla \widetilde{w}-\widetilde{\gamma}) \mathrm{d} \omega+\int_{\omega} \gamma \cdot \mathcal{C}^{\mathrm{s}} \cdot \widetilde{\gamma} \mathrm{d} \omega, \\
l(\widetilde{u}) & =\int_{\omega} p \widetilde{w} \mathrm{~d} \omega
\end{aligned}
$$

with the variables $u=\left[w, \gamma_{1}, \gamma_{2}\right]^{\top}$. First derivatives of $\gamma$ and second derivatives of $w$ call for $\gamma \in H^{1}(\omega)$ and $w \in H^{2}(\omega)$. Thus, a discretisation with $C^{0}$ elements for $\gamma$ and $C^{1}$ elements for $w$ is needed. The name "hierarchic" comes from the fact that the bilinear form includes the Kirchhoff bending term and can thus be viewed as a hierarchic extension of the Kirchhoff model.

Results for the example problem from section 2.1 are shown in Table 3. One observes good $h$-convergence to the right solutions for the thick and the thin plate. The hierarchic reformulation has rendered the problem locking free and the method performs well for thick and thin plates. There is, however, one major drawback: The iteration numbers needed to reduce the initial residual below the defined threshold are significantly higher for the thick plate than for the thin plate and also much higher than with the standard Mindlin-Reissner formulation. A fair and thorough comparison between the pure $C^{0}$ elements used there and the partially $C^{1}$ combined elements here also needs to involve some error and computation time measurement and is carried out in section 2.11. But the reduced comparison given here still serves to show that it might be useful to search for further formulations even though the hierarchic formulation is locking free. 


\begin{tabular}{rrrrr}
\hline & \multicolumn{2}{c}{ thick plate } & \multicolumn{2}{c}{ thin plate } \\
\#E1 & $w_{\max }$ & \#it & $w_{\max }$ & \#it \\
\hline 4 & $1.63 \mathrm{E}-2$ & 3 & $1.45 \mathrm{E}-2$ & 3 \\
16 & $1.56 \mathrm{E}-2$ & 34 & $1.38 \mathrm{E}-2$ & 14 \\
64 & $1.58 \mathrm{E}-2$ & 265 & $1.38 \mathrm{E}-2$ & 63 \\
256 & $1.59 \mathrm{E}-2$ & 910 & $1.38 \mathrm{E}-2$ & 204 \\
1024 & $1.60 \mathrm{E}-2$ & 2367 & $1.38 \mathrm{E}-2$ & 559 \\
4096 & $1.60 \mathrm{E}-2$ & 5000 & $1.38 \mathrm{E}-2$ & 1241 \\
16384 & $1.60 \mathrm{E}-2$ & 5000 & $1.38 \mathrm{E}-2$ & 2837 \\
\hline
\end{tabular}

Table 4: Results for the rotation free Mindlin-Reissner formulation with $C^{1}$ BFS elements for $w, w_{\mathrm{sb}}$ and $w_{\mathrm{bs}}$

\subsection{The Mindlin-Reissner plate in a rotation free formulation by Oesterle, Ramm and Bischoff (ORB)}

The locking phenomenon is caused by the imbalance of function values and derivative values in the standard formulation. The authors of [16] try to overcome this issue with a so-called rotation free formulation. All variables of this formulation represent displacements and not rotation angles. Two additional plate deflections whose derivatives represent the shear angles need to be introduced for an equivalent formulation to the Mindlin-Reissner plate. The ansatz

$$
w=w_{\mathrm{b}}+w_{\mathrm{sb}}+w_{\mathrm{bs}}, \gamma=\left(w_{\mathrm{sb}, 1}, w_{\mathrm{bs}, 2}\right)^{\top}, \theta=\nabla w-\gamma=\nabla w_{\mathrm{b}}+\left(w_{\mathrm{bs}, 1}, w_{\mathrm{sb}, 2}\right)^{\top}
$$

yields the formulation

$$
\begin{aligned}
u^{3 \mathrm{D}} & =\left[\begin{array}{c}
u_{1}-x_{3}\left(w_{\mathrm{b}, 1}+w_{\mathrm{bs}, 1}\right) \\
u_{2}-x_{3}\left(w_{\mathrm{b}, 2}+w_{\mathrm{sb}, 2}\right) \\
w
\end{array}\right], \\
a(u, \widetilde{u}) & =\int_{\omega} \varepsilon\left(\begin{array}{c}
w_{\mathrm{b}, 1}+w_{\mathrm{bs}, 1} \\
w_{\mathrm{b}, 2}+w_{\mathrm{sb}, 2}
\end{array}\right): \mathfrak{C}^{\mathrm{b}}: \varepsilon\left(\begin{array}{l}
\widetilde{w}_{\mathrm{b}, 1}+\widetilde{w}_{\mathrm{bs}, 1} \\
\widetilde{w}_{\mathrm{b}, 2}+\widetilde{w}_{\mathrm{sb}, 2}
\end{array}\right) \mathrm{d} \omega+\int_{\omega}\left(\begin{array}{c}
w_{\mathrm{sb}, 1} \\
w_{\mathrm{bs}, 2}
\end{array}\right) \cdot \mathcal{C}^{\mathrm{s}} \cdot\left(\begin{array}{c}
\widetilde{w}_{\mathrm{sb}, 1} \\
\widetilde{w}_{\mathrm{bs}, 2}
\end{array}\right) \mathrm{d} \omega, \\
l(\widetilde{u}) & =\int_{\omega} p\left(\widetilde{w}_{\mathrm{b}}+\widetilde{w}_{\mathrm{sb}}+\widetilde{w}_{\mathrm{bs}}\right) \mathrm{d} \omega .
\end{aligned}
$$

All three variables $w_{\mathrm{b}}, w_{\mathrm{sb}}, w_{\mathrm{bs}}$ are present with second derivatives and need to be discretised with $C^{1}$ elements. Unlike the source [16] which uses isogeometric analysis we again employ standard $C^{1}$ finite elements.

Results for the example problem from section 2.1 are shown in Table 4 . The conjugate gradient method was stopped if the stopping criterion was not met after 5000 iterations. One observes good $h$-convergence to the right solutions for the thick and the thin plate. The rotation free reformulation has rendered the problem locking free and the method performs well for thick and thin plates. The iteration numbers, however, are extremely high in comparison with the other methods. With shear locking gone but very low 


\begin{tabular}{rrrrr}
\hline & \multicolumn{2}{c}{ thick plate } & \multicolumn{2}{c}{ thin plate } \\
\#E1 & $w_{\max }$ & \#it & $w_{\max }$ & \#it \\
\hline 4 & $1.63 \mathrm{E}-2$ & 3 & $1.45 \mathrm{E}-2$ & 3 \\
16 & $1.55 \mathrm{E}-2$ & 24 & $1.38 \mathrm{E}-2$ & 119 \\
64 & $1.56 \mathrm{E}-2$ & 49 & $1.38 \mathrm{E}-2$ & 1320 \\
256 & $1.57 \mathrm{E}-2$ & 96 & $1.38 \mathrm{E}-2$ & 3132 \\
1024 & $1.57 \mathrm{E}-2$ & 103 & $1.38 \mathrm{E}-2$ & 2666 \\
4096 & $1.57 \mathrm{E}-2$ & 139 & $1.38 \mathrm{E}-2$ & 834 \\
16384 & $1.57 \mathrm{E}-2$ & 142 & $1.38 \mathrm{E}-2$ & 483 \\
\hline
\end{tabular}

Table 5: Results for the Endo-Kimura plate formulation with $C^{1}$ BFS elements for $w$ and $w_{\mathrm{b}}$

numerical efficiency this method does not look promising for practical use. Maybe there exist better solvers for this problem formulation, but at least our preconditioned solver is inefficient in this case.

\subsection{The plate formulation of Endo and Kimura (EKs)}

The authors of [9] argue that the bending and shear deformations of the Mindlin-Reissner plate formulation can not be determined uniquely and therefore propose a different formulation. Like in the standard formulation they eliminate $\gamma=\nabla w-\theta$ from the three variable formulation (2)-(5) and make the additional assumption $\theta=\nabla w_{\mathrm{b}}$ for some $w_{\mathrm{b}}$. In consequence it holds $\gamma=\nabla w_{\mathrm{s}}$ for $w_{\mathrm{s}}=w-w_{\mathrm{b}}$. Just like the formulation of the previous section the absence of rotation angle variables leads to a rotation free and thus also locking free formulation. One gets

$$
\begin{aligned}
u^{3 \mathrm{D}} & =\left[\begin{array}{c}
u_{1}-x_{3} w_{\mathrm{b}, 1} \\
u_{2}-x_{3} w_{\mathrm{b}, 2} \\
w
\end{array}\right], \\
a(u, \widetilde{u}) & =\int_{\omega} \varepsilon\left(\nabla w_{\mathrm{b}}\right): \mathfrak{C}^{\mathrm{b}}: \varepsilon\left(\nabla \widetilde{w}_{\mathrm{b}}\right) \mathrm{d} \omega+\int_{\omega}\left(\nabla w-\nabla w_{\mathrm{b}}\right) \cdot \mathcal{C}^{\mathrm{s}} \cdot\left(\nabla \widetilde{w}-\nabla \widetilde{w}_{\mathrm{b}}\right) \mathrm{d} \omega, \\
l(\widetilde{u}) & =\int_{\omega} p \widetilde{w} \mathrm{~d} \omega .
\end{aligned}
$$

Both variables $w$ and $w_{\mathrm{b}}$ are present with second derivatives and need to be discretised with $C^{1}$ elements. One expects a slightly different solution than with the standard Mindlin-Reissner plate due to the additional condition that $\theta$ needs to be a gradient.

Numerical results are shown in Table 5 . One observes good $h$-convergence for both the thick and the thin plate. The solution of the thick plate is different from the MindlinReissner plate. The additional gradient condition leads to a slightly stiffer behaviour. It is unclear which solution is the "better" one compared to the behaviour of real plates. Iteration numbers are good for the thin plate but very high for the thick plate. 


\begin{tabular}{rrrrr}
\hline & \multicolumn{2}{c}{ thick plate } & \multicolumn{2}{c}{ thin plate } \\
\#E1 & $w_{\max }$ & \#it & $w_{\max }$ & \#it \\
\hline 4 & $1.63 \mathrm{E}-2$ & 3 & $1.45 \mathrm{E}-2$ & 3 \\
16 & $1.55 \mathrm{E}-2$ & 17 & $1.38 \mathrm{E}-2$ & 9 \\
64 & $1.56 \mathrm{E}-2$ & 103 & $1.38 \mathrm{E}-2$ & 28 \\
256 & $1.57 \mathrm{E}-2$ & 374 & $1.38 \mathrm{E}-2$ & 48 \\
1024 & $1.57 \mathrm{E}-2$ & 706 & $1.38 \mathrm{E}-2$ & 59 \\
4096 & $1.57 \mathrm{E}-2$ & 1383 & $1.38 \mathrm{E}-2$ & 100 \\
16384 & $1.57 \mathrm{E}-2$ & 2829 & $1.38 \mathrm{E}-2$ & 139 \\
\hline
\end{tabular}

Table 6: Results for the hierarchic Endo-Kimura plate formulation with $C^{1}$ BFS elements for $w$ and $w_{\mathrm{s}}$

\subsection{The Endo-Kimura plate in hierarchic formulation (EKh)}

Endo and Kimura also suggested that their ansatz $\theta=\nabla w_{\mathrm{b}}, \gamma=w_{\mathrm{s}}$ can be combined with the hierarchic ansatz of eliminating $\theta$ instead of $\gamma$ from the three variable formulation (2)-(5). This yields

$$
\begin{aligned}
u^{3 \mathrm{D}} & =\left[\begin{array}{c}
u_{1}-x_{3}\left(w_{, 1}-w_{\mathrm{s}, 1}\right) \\
u_{2}-x_{3}\left(w_{, 2}-w_{\mathrm{s}, 2}\right) \\
w
\end{array}\right], \\
a(u, \widetilde{u}) & =\int_{\omega} \varepsilon\left(\nabla w-\nabla w_{\mathrm{s}}\right): \mathfrak{C}^{\mathrm{b}}: \varepsilon\left(\nabla \widetilde{w}-\nabla \widetilde{w}_{\mathrm{s}}\right) \mathrm{d} \omega+\int_{\omega}\left(\nabla w_{\mathrm{s}}\right) \cdot \mathcal{C}^{\mathrm{s}} \cdot\left(\nabla \widetilde{w}_{\mathrm{s}}\right) \mathrm{d} \omega, \\
l(\widetilde{u}) & =\int_{\omega} p \widetilde{w} \mathrm{~d} \omega .
\end{aligned}
$$

The numerical results in Table 6 show good $h$-convergence to the same solution like the standard Endo-Kimura formulation. Iteration numbers are good for the thick plate but very high for the thin plate. This is exactly the other way around than with standard Endo-Kimura in the previous section. It appears that the difficulty of the problem has shifted from the thick to the thin plate, or from the shear to the bending term.

\subsection{First new formulation: Endo-Kimura plate decoupled (EKd)}

Another kind of elimination which was not present in [9] is possible. With the ansatz of Endo and Kimura one can also eliminate $\nabla w=\nabla w_{\mathrm{b}}+\nabla w_{\mathrm{s}}$ and $w=w_{\mathrm{b}}+w_{\mathrm{s}}$ from the 


\begin{tabular}{rcrrr}
\hline & \multicolumn{2}{c}{ thick plate } & \multicolumn{2}{c}{ thin plate } \\
\#E1 & $w_{\max }$ & $\#$ it & $w_{\max }$ & \#it \\
\hline 4 & $1.69 \mathrm{E}-2$ & 2 & $1.45 \mathrm{E}-2$ & 2 \\
16 & $1.58 \mathrm{E}-2$ & 10 & $1.38 \mathrm{E}-2$ & 9 \\
64 & $1.58 \mathrm{E}-2$ & 22 & $1.38 \mathrm{E}-2$ & 22 \\
256 & $1.57 \mathrm{E}-2$ & 26 & $1.38 \mathrm{E}-2$ & 35 \\
1024 & $1.57 \mathrm{E}-2$ & 24 & $1.38 \mathrm{E}-2$ & 29 \\
4096 & $1.57 \mathrm{E}-2$ & 21 & $1.38 \mathrm{E}-2$ & 29 \\
16384 & $1.57 \mathrm{E}-2$ & 20 & $1.38 \mathrm{E}-2$ & 33 \\
\hline
\end{tabular}

Table 7: Results for the decoupled Endo-Kimura plate formulation with $C^{1}$ BFS elements for $w_{\mathrm{b}}$ and $C^{0}$ bilinear elements for $w_{\mathrm{s}}$

equations. Only the variables $w_{\mathrm{b}}$ and $w_{\mathrm{s}}$ remain and one arrives at

$$
\begin{aligned}
u^{3 \mathrm{D}} & =\left[\begin{array}{c}
u_{1}-x_{3} w_{\mathrm{b}, 1} \\
u_{2}-x_{3} w_{\mathrm{b}, 2} \\
w
\end{array}\right], \\
a(u, \widetilde{u}) & =\int_{\omega} \varepsilon\left(\nabla w_{\mathrm{b}}\right): \mathfrak{C}^{\mathrm{b}}: \varepsilon\left(\nabla \widetilde{w}_{\mathrm{b}}\right) \mathrm{d} \omega+\int_{\omega}\left(\nabla w_{\mathrm{s}}\right) \cdot \mathcal{C}^{\mathrm{s}} \cdot\left(\nabla \widetilde{w}_{\mathrm{s}}\right) \mathrm{d} \omega, \\
l(\widetilde{u}) & =\int_{\omega} p\left(\widetilde{w}_{\mathrm{b}}+\widetilde{w}_{\mathrm{s}}\right) \mathrm{d} \omega .
\end{aligned}
$$

This formulation has completely decoupled bending and shear terms. The bending deflection $w_{\mathrm{b}}$ is the same as in the Kirchhoff theory and an additional shear deflection $w_{\mathrm{s}}$ may be calculated separately and added for a total deflection $w=w_{\mathrm{b}}+w_{\mathrm{s}}$ afterwards. We solve, however, the complete system. An analogous decoupled formulation is not directly obtainable from the standard Mindlin-Reissner formulation. A conforming discretisation can be achieved with $C^{1}$ elements for $w_{\mathrm{b}}$ and $C^{0}$ elements for $w_{\mathrm{s}}$.

The numerical results in Table 7 show good $h$-convergence to the same solution like the standard Endo-Kimura formulation. Iteration numbers are very good for both the thick and the thin plate.

The decoupling has eliminated the convergence problems of the other two Endo-Kimura formulations. We have obtained a formulation which is suitable for both thick and thin plates and is numerically efficient in both cases. The only drawback is that we do not get the solution of the Mindlin-Reissner plate but of a slightly stiffer problem.

\subsection{Second new formulation: hierarchic Mindlin-Reissner based on Endo-Kimura (MREK)}

We propose to extend the formulation of the previous section with an additional term to relax the gradient condition again. In this way we arrive at a Mindlin-Reissner 


\begin{tabular}{rrrrr}
\hline & \multicolumn{2}{c}{ thick plate } & \multicolumn{2}{c}{ thin plate } \\
\#E1 & $w_{\max }$ & \#it & $w_{\max }$ & \#it \\
\hline 4 & $1.69 \mathrm{E}-2$ & 2 & $1.45 \mathrm{E}-2$ & 2 \\
16 & $1.60 \mathrm{E}-2$ & 9 & $1.38 \mathrm{E}-2$ & 10 \\
64 & $1.60 \mathrm{E}-2$ & 26 & $1.38 \mathrm{E}-2$ & 25 \\
256 & $1.60 \mathrm{E}-2$ & 46 & $1.38 \mathrm{E}-2$ & 50 \\
1024 & $1.60 \mathrm{E}-2$ & 60 & $1.38 \mathrm{E}-2$ & 68 \\
4096 & $1.60 \mathrm{E}-2$ & 100 & $1.38 \mathrm{E}-2$ & 100 \\
16384 & $1.60 \mathrm{E}-2$ & 130 & $1.38 \mathrm{E}-2$ & 132 \\
\hline
\end{tabular}

Table 8: Results for the Endo-Kimura based Mindlin-Reissner plate formulation with $C^{1}$ BFS elements for $w_{\mathrm{b}}$ and $C^{0}$ bilinear elements for $w_{\mathrm{s}}$ and $\rho$

formulation which can be viewed as hierarchically based on the decoupled Endo-Kimura formulation. The ansatz

$$
w=w_{\mathrm{b}}+w_{\mathrm{s}}, \quad \nabla w=\theta+\gamma, \quad \theta=\nabla w_{\mathrm{b}}-\rho, \quad \gamma=\nabla w_{\mathrm{s}}+\rho
$$

and elimination of $w$ yields

$$
\begin{aligned}
u^{3 \mathrm{D}} & =\left[\begin{array}{c}
u_{1}-x_{3}\left(w_{\mathrm{b}, 1}-\rho_{1}\right) \\
u_{2}-x_{3}\left(w_{\mathrm{b}, 2}-\rho_{2}\right) \\
w
\end{array}\right], \\
a(u, \widetilde{u}) & =\int_{\omega} \varepsilon\left(\nabla w_{\mathrm{b}}-\rho\right): \mathfrak{C}^{\mathrm{b}}: \varepsilon\left(\nabla \widetilde{w}_{\mathrm{b}}-\widetilde{\rho}\right) \mathrm{d} \omega+\int_{\omega}\left(\nabla w_{\mathrm{s}}+\rho\right) \cdot \mathcal{C}^{\mathrm{s}} \cdot\left(\nabla \widetilde{w}_{\mathrm{s}}+\widetilde{\rho}\right) \mathrm{d} \omega, \\
l(\widetilde{u}) & =\int_{\omega} p\left(\widetilde{w}_{\mathrm{b}}+\widetilde{w}_{\mathrm{s}}\right) \mathrm{d} \omega .
\end{aligned}
$$

A conforming discretisation can be achieved with $C^{1}$ elements for $w_{\mathrm{b}}$ and $C^{0}$ elements for $\rho_{1}, \rho_{2}$ and $w_{\mathrm{s}}$.

The numerical results in Table 8 show good $h$-convergence to the same solution like for the Mindlin-Reissner model. Iteration numbers are not as good as for the decoupled Endo-Kimura formulation but still good. They are comparable for the thick and thin plate and grow slowly with the number of elements. In comparison with the hierarchic Mindlin-Reissner formulation from 2.5, the iteration numbers for the thin plate are slightly bigger and those of the thick plate are somewhat higher at lower element numbers but are growing slower and thus they are lower than those of the hierarchic MindlinReissner formulation at higher element numbers.

\subsection{Comparison of numerical results}

The numerical example from section 2.1 is explored in more detail in this section. We consider the error estimator of [4] for MITC elements and neglect the terms which are 


\begin{tabular}{lllcl}
\hline formulation & $C^{0}$ unkn. & $C^{1}$ unkn. & dofs/node (rectangle) & dofs/node (triangle) \\
\hline MRs & 3 & 0 & 3 & 3 \\
MRh & 2 & 1 & 6 & 5 \\
ORB & 0 & 3 & 12 & 9 \\
EKs & 0 & 2 & 8 & 6 \\
EKh & 0 & 2 & 8 & 6 \\
EKd & 1 & 1 & 5 & 4 \\
MREK & 3 & 1 & 7 & 6 \\
\hline
\end{tabular}

Table 9: Continuous and discretised unknowns of the plate formulations

zero for our standard elements. The error contribution of an element $T$ then reads

$$
\begin{aligned}
\eta_{T}^{2}= & h_{T}^{2}\left(\left\|\operatorname{div}\left(\mathfrak{C}^{\mathrm{b}}: \varepsilon\left(\theta_{h}\right)\right)+\mathcal{C}^{\mathrm{s}} \cdot \gamma_{h}\right\|_{L^{2}(T)}+\left(d^{2}+h_{T}^{2}\right)\left\|p+\operatorname{div}\left(\mathcal{C}^{\mathrm{s}} \cdot \gamma_{h}\right)\right\|_{L^{2}(T)}\right) \\
& +\frac{1}{2} \sum_{E \in \mathcal{E}(T)} h_{E}\left(\left\|\left[\mathfrak{C}^{\mathrm{b}}: \varepsilon\left(\theta_{h}\right)\right]_{E} \cdot \boldsymbol{n}_{E}\right\|_{L^{2}(E)}+\left(d^{2}+h_{E}^{2}\right)\left\|\left[\mathcal{C}^{\mathrm{s}} \cdot \gamma_{h}\right]_{E} \cdot \boldsymbol{n}_{E}\right\|_{L^{2}(E)}\right)
\end{aligned}
$$

for a finite element solution $\theta_{h}$ and $\gamma_{h}$ (adapted to the actual used theory for each of the Mindlin-Reissner and Endo-Kimura plate formulations). The formula involves the element diameter $h_{T}$, the set of edges $\mathcal{E}(T)$ of the element $T$, the edge length $h_{E}$, the jump $[\bullet]_{E}$ of a quantity over the edge $E$ and a fixed unit normal $\boldsymbol{n}_{E}$ of edge $E$.

Computations were done with our TU Chemnitz adaptive FEM software SPC written in Fortran, see [12]. The software features adaptive FEM but uniform refinement was employed for this tests for good comparability. The plate module of SPC features rectangular and triangular elements. The coarse mesh for our example with rectangular elements is one unit square; the coarse mesh for triangular elements is the unit square divided by one of its diagonals. Square element use Bogner-Fox-Schmit (BFS) quadrangles for $C^{1}$ parts and Lagrange elements for $C^{0}$ parts of the different formulations as detailed in the sections above. Triangular elements use reduced Hsieh-Clough-Tocher (rHCT) elements for $C^{1}$ parts and Lagrange elements for $C^{0}$ parts of the different formulations as detailed in the sections above. BFS elements feature 4 degrees of freedom (dofs) per node and unknown in the ansatz, rHCT elements 3 dofs per node and unknown. This leads to unknowns per node as given in Table 9.

Figure 3 collects the development of the error estimator for the thick and thin plates in case of square elements. The first row shows its reduction over the number of elements. All formulations show $h$-convergence with best results for MREK and EKd for the thick plate and MREK for the thin plate.

The second row shows the error reduction over the total number of unknowns. The unknowns are approximately linked to the element numbers by a fixed factor because of the uniform refinement. Thus, the graphs do look quite similar for both the thick and the thin plate compared to those of the first row. 
The third row shows the error reduction over the total time summed over all refinements. These two are the most important graphs because only they factor in the iteration numbers via the computation time. EKd is best closely followed by MREK and they are clearly more efficient than the other formulations for the thick plate. For the thin plate this changes to MREK as the most efficient formulation followed by EKd and than the other formulations.

The results for triangular elements are collected in Figure 4. The performance differences are less pronounced in this case than in the square elements case. EKd is the most efficient formulation for both the thick and the thin plate. MREK, EKh and MRh are also performing quite well in both cases. EKs is very good for thick plates but rather inefficient for the thin plate.

In summary, MREK is the most efficient Mindlin-Reissner formulation and EKd the most efficient Endo-Kimura formulation for thick and thin as well as square and triangular elements. We conclude that both our new formulations are the formulations of choice for flexibly usable thick and thin Mindlin-Reissner or Endo-Kimura plate elements. The MRh formulation comes close in performance to MREK in case of triangular elements but is clearly outperformed by MREK in case of square elements. 
thick plate
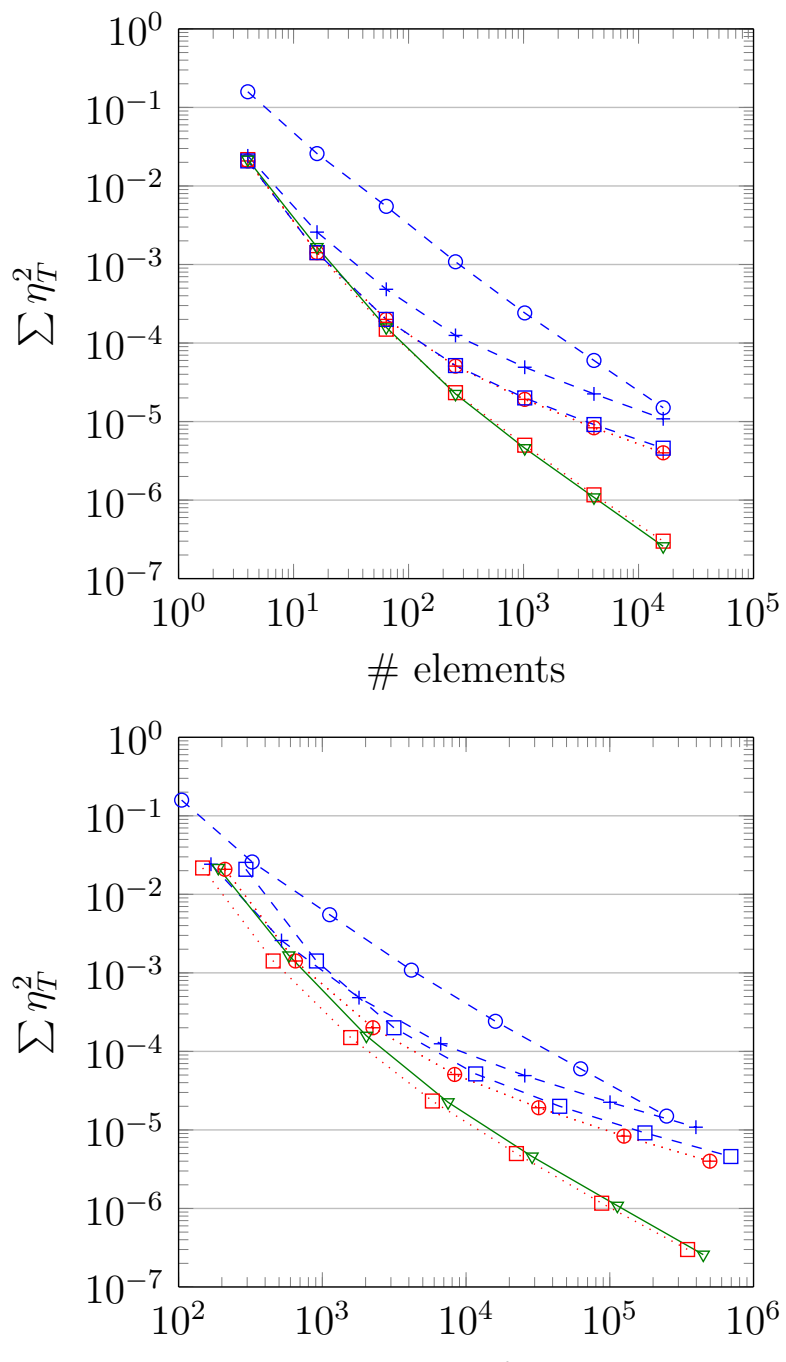

\# dofs

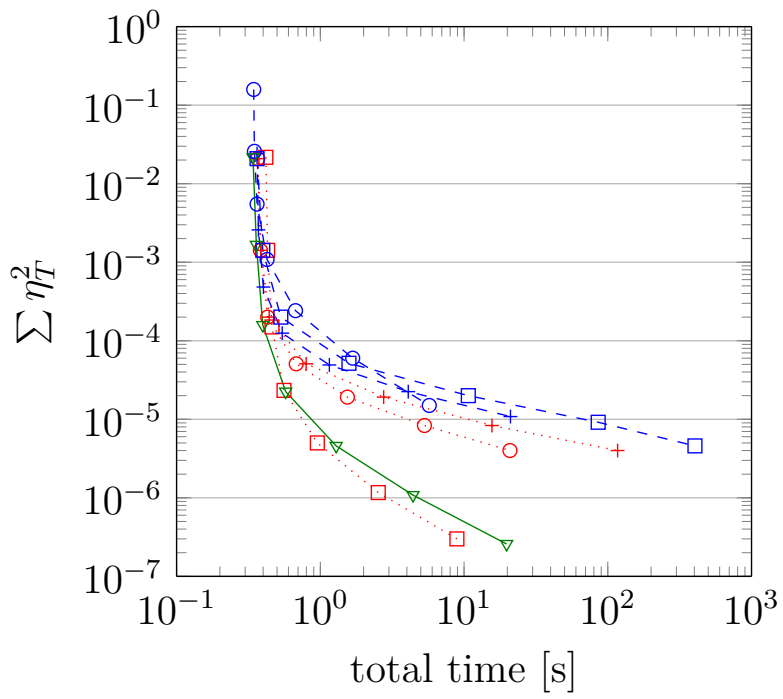

thin plate
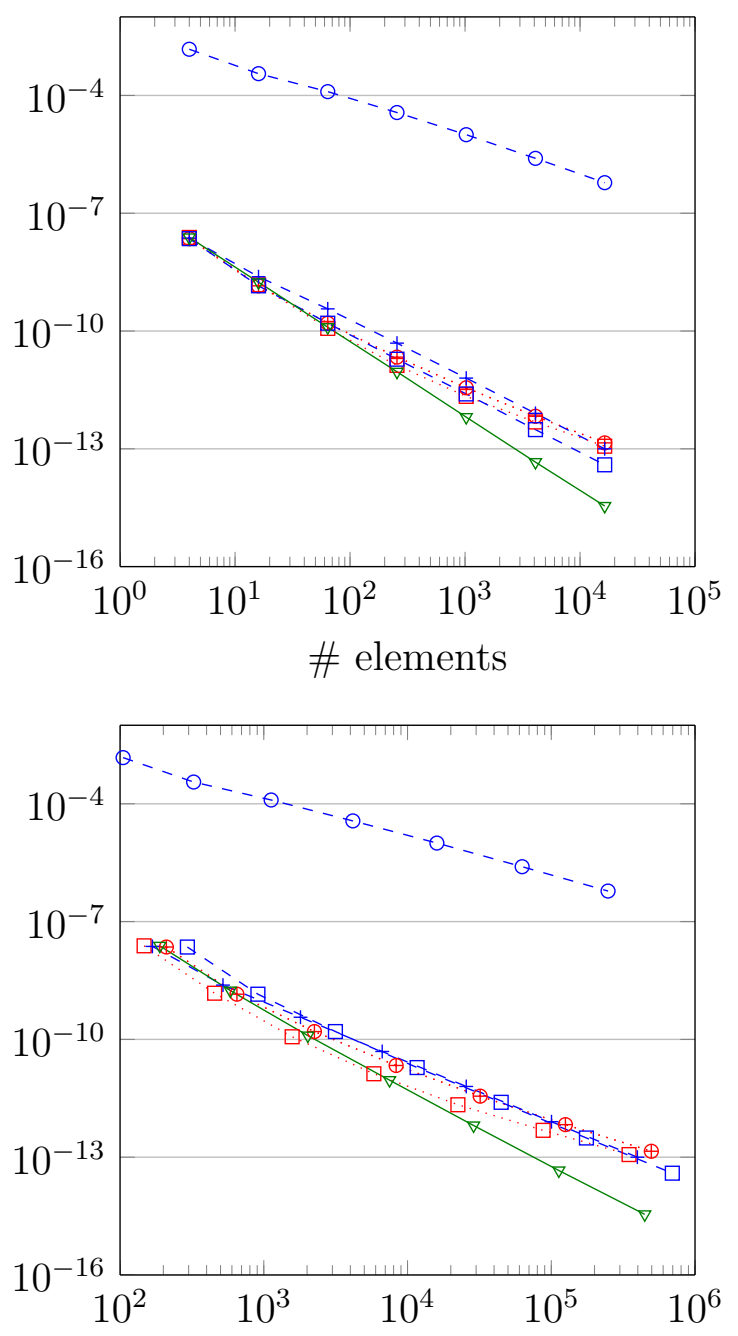

\# dofs

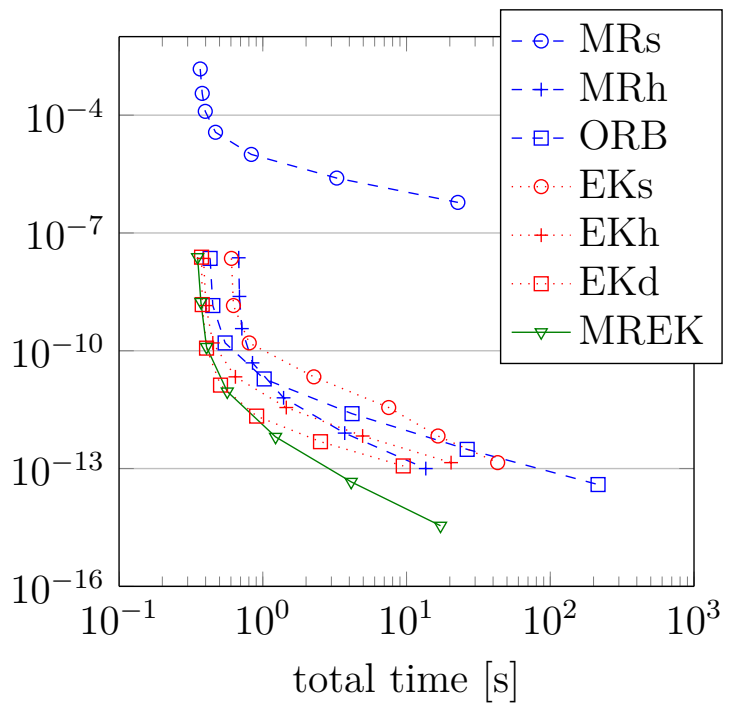

Figure 3: Development of squared relative error estimator for square elements 
thick plate
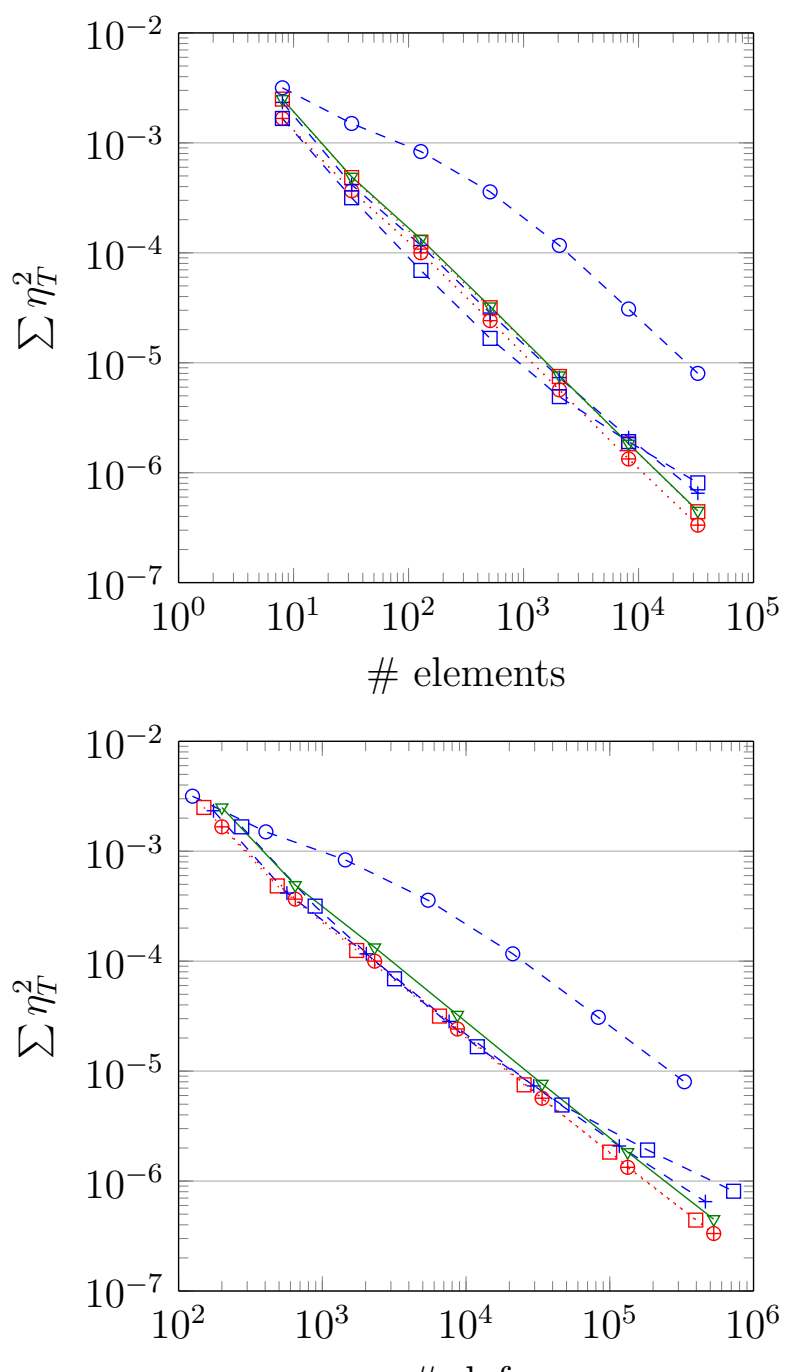

\# dofs

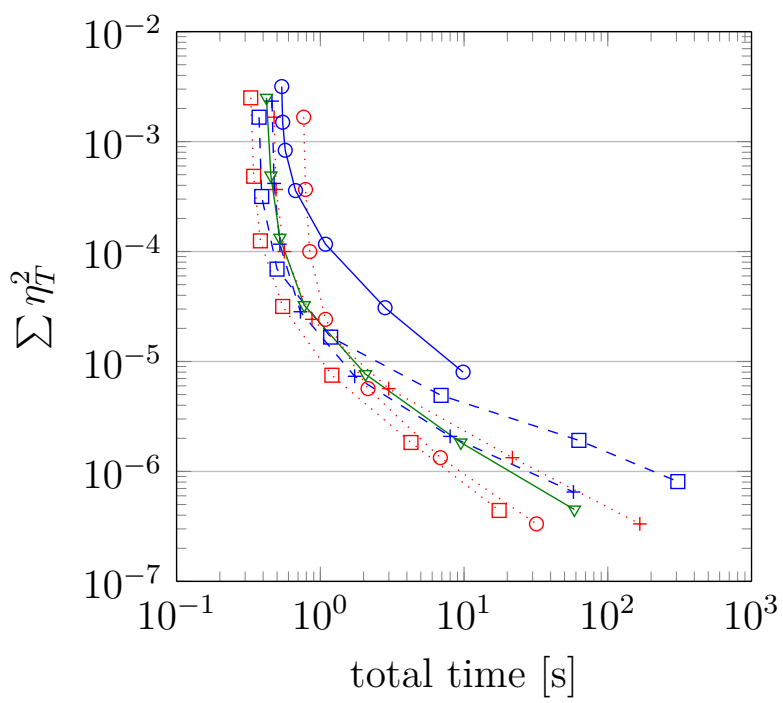

thin plate
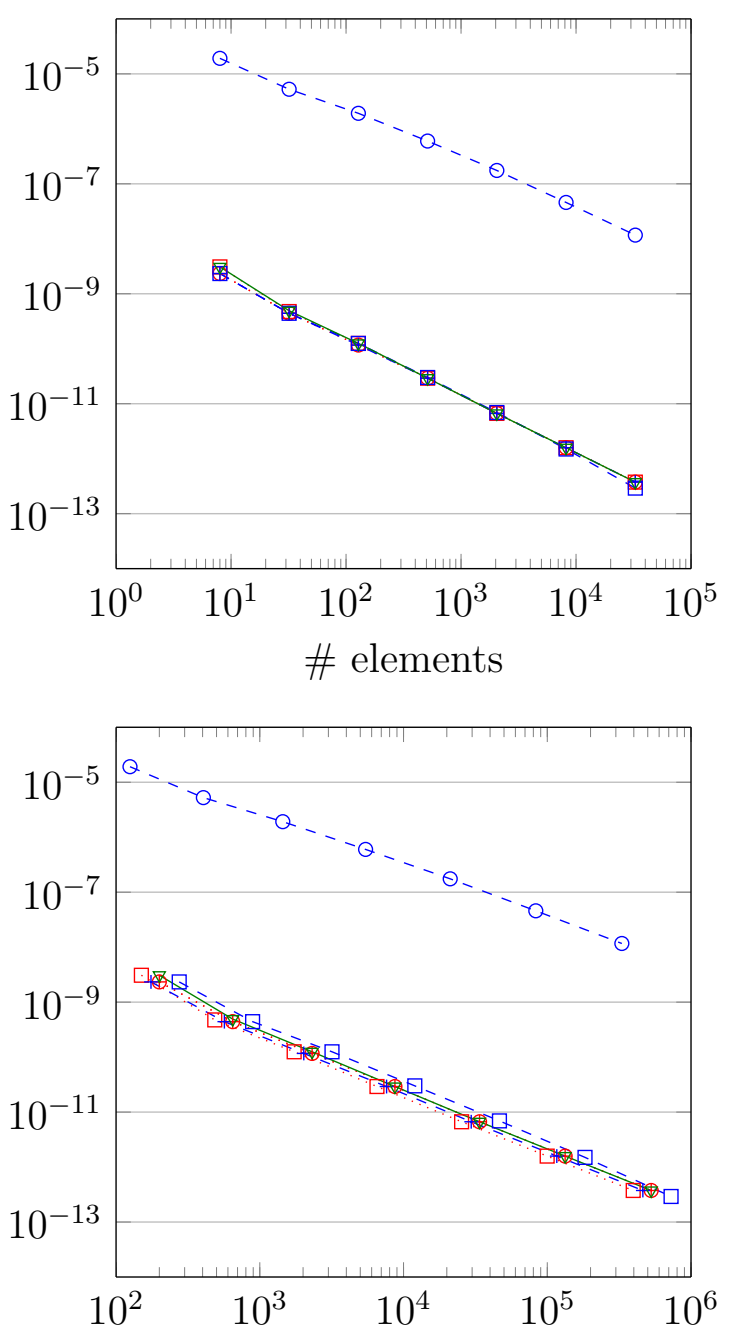

\# dofs

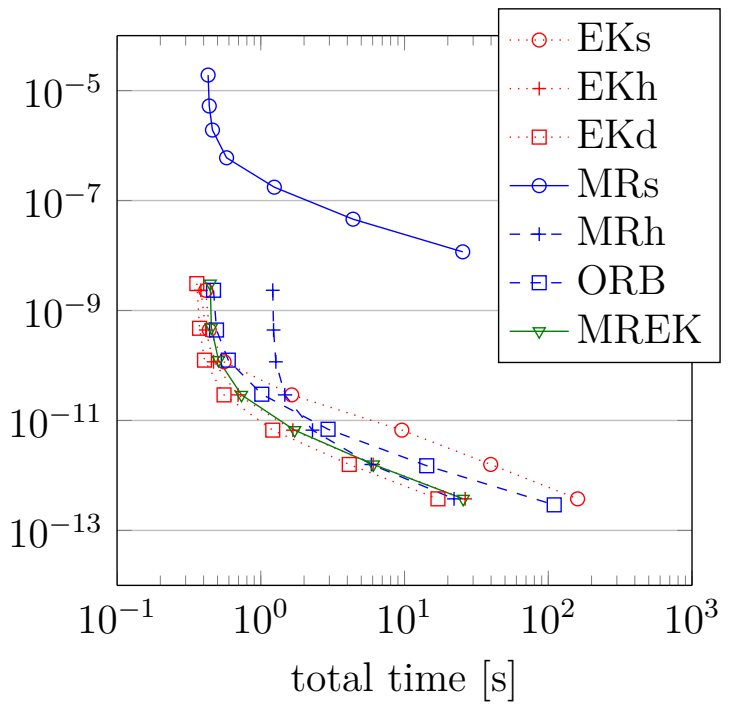

Figure 4: Development of squared relative error estimator for triangle elements 


\section{Shell theory}

\subsection{The Naghdi shell}

We consider a shell, a thin-walled, possibly curved structure in 3D. The shell domain is defined by the midsurface $\boldsymbol{y}$ and the thickness $d$ (significantly smaller than the midsurface dimensions) via

$$
\left\{\boldsymbol{x}\left(\eta^{1}, \eta^{2}, \tau\right)=\boldsymbol{y}\left(\eta^{1}, \eta^{2}\right)+\tau \boldsymbol{a}_{3}\left(\eta^{1}, \eta^{2}\right):\left(\eta^{1}, \eta^{2}\right) \in \omega \subset \mathbb{R}^{2}, \tau \in\left[-\frac{d}{2}, \frac{d}{2}\right]\right\}
$$

with the tangential vectors $\boldsymbol{a}_{1}, \boldsymbol{a}_{2}$ and the unit normal vector $\boldsymbol{a}_{3}$ of the midsurface given by

$$
\boldsymbol{a}_{i}=\boldsymbol{y}_{, i}=\frac{\partial \boldsymbol{y}}{\partial \eta^{i}}, i=1,2, \quad \boldsymbol{a}_{3}=\frac{\boldsymbol{a}_{1} \times \boldsymbol{a}_{2}}{\left\|\boldsymbol{a}_{1} \times \boldsymbol{a}_{2}\right\|} .
$$

These vectors are called covariant basis and form a biorthogonal system with the contravariant basis $\boldsymbol{a}^{1}, \boldsymbol{a}^{2}$ and $\boldsymbol{a}^{3}=\boldsymbol{a}_{3}$. We abbreviate the parameter derivative $\partial \bullet / \partial \eta^{i}$ of any object $\bullet$ with an index $\bullet, i$ throughout this section.

The solution of a shell deformation problem with 3D-FEM is not suitable due to the small thickness (either elements with bad aspect ratios occur or an extreme amount of elements is required). Therefore, special shell models are needed. One popular example is the Naghdi shell model, see for example [5, section 4.2.2] and the references therein. It employs the Mindlin-Reissner hypotheses (normal lines remain straight after deformation, no change of thickness, plane state of stress) with the deformation ansatz

$$
\boldsymbol{u}^{3 \mathrm{D}}:=\boldsymbol{u}\left(\eta^{1}, \eta^{2}\right)+\tau \boldsymbol{\theta}\left(\eta^{1}, \eta^{2}\right) \quad \text { with } \quad \boldsymbol{u}=u_{1} \boldsymbol{a}^{1}+u_{2} \boldsymbol{a}^{2}+u_{3} \boldsymbol{a}_{3}, \boldsymbol{\theta}=\theta_{1} \boldsymbol{a}^{1}+\theta_{2} \boldsymbol{a}^{2}
$$

in covariant coordinates with the midsurface translation $\boldsymbol{u}$ and the rotation $\boldsymbol{\theta}$.

\subsubsection{Naghdi shell in covariant coordinates}

We collect the five unknown covariant coordinates in a vector

$$
u=\left[u_{1}, u_{2}, u_{3}, \theta_{1}, \theta_{2}\right]^{\top} .
$$

The weak formulation of the shell problem reads

$$
a(u, \widetilde{u})=f(\widetilde{u}) \quad \forall \widetilde{u}
$$

with test functions $\widetilde{u}$ from an appropriate space, the bilinear form $a(\cdot, \cdot)$ accounting for the inner virtual work of the elastic deformation and the linear functional $f(\cdot)$ accounting 
for the load of the test functions. The bilinear form can be written as a sum of membrane, bending and shear terms with according strains in the form

$$
\begin{aligned}
a(u, \widetilde{u}) & =a^{\mathrm{m}}(u, \widetilde{u})+a^{\mathrm{b}}(u, \widetilde{u})+a^{\mathrm{s}}(u, \widetilde{u}), \\
a^{\mathrm{m}}(u, \widetilde{u}) & =\int_{\omega} \varepsilon^{\mathrm{m}}(u): \mathfrak{C}^{\mathrm{m}}: \varepsilon^{\mathrm{m}}(\widetilde{u}) \mathrm{d} \mathcal{S} \\
a^{\mathrm{b}}(u, \widetilde{u}) & =\int_{\omega} \varepsilon^{\mathrm{b}}(u): \mathfrak{C}^{\mathrm{b}}: \varepsilon^{\mathrm{b}}(\widetilde{u}) \mathrm{d} \mathcal{S} \\
a^{\mathrm{s}}(u, \widetilde{u}) & =\int_{\omega} \varepsilon^{\mathrm{s}}(u): \mathfrak{C}^{\mathrm{m}}: \varepsilon^{\mathrm{s}}(\widetilde{u}) \mathrm{d} \mathcal{S}
\end{aligned}
$$

with the surface element $\mathrm{d} \mathcal{S}=\left\|\boldsymbol{a}_{1} \times \boldsymbol{a}_{2}\right\| \mathrm{d} \eta^{1} \mathrm{~d} \eta^{2}$. The membrane, bending and shear strain tensors

$$
\boldsymbol{\varepsilon}^{\mathrm{m}}(u)=\sum_{i j} \varepsilon_{i j}^{\mathrm{m}}(u) \boldsymbol{a}^{i} \boldsymbol{a}^{j}, \boldsymbol{\varepsilon}^{\mathrm{b}}(u)=\sum_{i j} \varepsilon_{i j}^{\mathrm{b}}(u) \boldsymbol{a}^{i} \boldsymbol{a}^{j}, \boldsymbol{\varepsilon}^{\mathrm{s}}(u)=\Sigma_{i} \varepsilon_{i}^{\mathrm{s}}(u)\left(\boldsymbol{a}^{i} \boldsymbol{a}_{3}+\boldsymbol{a}_{3} \boldsymbol{a}^{i}\right)
$$

have the covariant coordinates

$$
\begin{aligned}
\varepsilon_{i j}^{\mathrm{m}}(u) & =\frac{1}{2}\left(u_{i \mid j}+u_{j \mid i}\right)-b_{i j} u_{3} \quad\left(\text { not dependent on } \theta_{i}\right), \\
\varepsilon_{i j}^{\mathrm{b}}(u) & =\frac{1}{2}\left(\theta_{i \mid j}+\theta_{j \mid i}-\Sigma_{k} b_{i}^{k} u_{k \mid j}-\Sigma_{k} b_{j}^{k} u_{k \mid i}\right)+c_{i j} u_{3}, \\
\varepsilon_{i}^{\mathrm{s}}(u) & =\frac{1}{2}\left(\theta_{i}+u_{3, i}+\Sigma_{j} b_{i}^{j} u_{j}\right) .
\end{aligned}
$$

The notation above uses the first fundamental form $a_{i j}=\boldsymbol{a}_{i} \cdot \boldsymbol{a}_{j}$ or $a^{i j}=\boldsymbol{a}^{i} \cdot \boldsymbol{a}^{j}$, the second fundamental form $b_{i j}=\boldsymbol{a}_{i, j} \cdot \boldsymbol{a}_{3}=-\boldsymbol{a}_{i} \cdot \boldsymbol{a}_{3, j}$ or $b_{i}^{j}=\Sigma_{k} b_{i k} a^{k j}$, the third fundamental form $c_{i j}=\Sigma_{l} b_{i}^{l} b_{l j}=\Sigma_{k l} b_{i k} a^{k l} b_{l j}$ and the covariant derivative $u_{i \mid j}=u_{i, j}-\Sigma_{k} \Gamma_{i j}^{k} u_{k}$ with the Christoffel symbols $\Gamma_{i j}^{k}=\boldsymbol{a}_{i, j} \cdot \boldsymbol{a}^{k}=\Sigma_{l} a^{k l}\left(a_{i l, j}+a_{j l, i}-a_{i j, l}\right) / 2$. Indices take the values 1 and 2 whenever a (multi-)sum $\Sigma$ without an index range appears. The membrane and bending material tensors $\mathfrak{C}^{\mathrm{m}}$ and $\mathfrak{C}^{\mathrm{b}}$ are obtained by integrating the material tensor $\mathfrak{C}$ and $\tau^{2} \mathfrak{C}$, respectively, over the thickness of the shell. If the material is constant over the thickness this leads to $\mathfrak{C}^{\mathrm{m}}=d \mathfrak{C}, \mathfrak{C}^{\mathrm{b}}=\frac{d^{3}}{12} \mathfrak{C}$. For a complete derivation of the above formulas we again refer to [5].

The action of the second order tensor $\varepsilon^{\mathrm{S}}(u)=\Sigma_{i} \varepsilon_{i}^{\mathrm{s}}(u)\left(\boldsymbol{a}^{i} \boldsymbol{a}_{3}+\boldsymbol{a}_{3} \boldsymbol{a}^{i}\right)$ with the fourth order material tensor $\mathfrak{C}^{\mathrm{m}}$ may be expressed by a replacement first order tensor $2 \boldsymbol{e}^{\mathrm{s}}(u)=$ $\Sigma_{i} \varepsilon_{i}^{\mathrm{s}}(u) \boldsymbol{a}^{i}$ with an appropriately chosen reduced second order shear tensor $\mathcal{C}^{\mathrm{s}}$ which reads

$$
a^{\mathrm{s}}(u, \widetilde{u})=\int_{\omega} \boldsymbol{e}^{\mathrm{s}}(u) \cdot \mathcal{C}^{\mathrm{s}} \cdot \boldsymbol{e}^{\mathrm{s}}(\widetilde{u}) \mathrm{d} \mathcal{S} .
$$

The shell midsurface can be given as a non-uniform rational B-spline (NURBS) surface for practical applications like outlined in [6] and [18].

\subsubsection{Coordinate free Naghdi shell formulation}

The traditional Naghdi shell formulation of the previous section uses the covariant coordinates as unknowns. The shell formulation itself can also be written in a coordinate 
free formulation which is independent of a coordinate system for the unknowns. With the surface gradient $\nabla_{\mathcal{S}}$ defined by

$$
\nabla_{\mathcal{S}} \bullet=\Sigma_{i} \boldsymbol{a}^{i} \bullet{ }_{, i}
$$

the equivalent of the angle decomposition (1) from plate theory reads $\nabla_{\mathcal{S}} \boldsymbol{u} \cdot \boldsymbol{a}_{3}=\boldsymbol{\theta}+\boldsymbol{\gamma}$. With the first and second fundamental tensors $\mathcal{A}=a_{i j} \boldsymbol{a}^{i} \boldsymbol{a}^{j}$ and $\mathcal{B}=b_{i j} \boldsymbol{a}^{i} \boldsymbol{a}^{j}$ the shell strains may then be expressed by

$$
\begin{aligned}
2 \varepsilon^{\mathrm{m}} & =\nabla_{\mathcal{S}} \boldsymbol{u} \cdot \mathcal{A}+\mathcal{A} \cdot\left(\nabla_{\mathcal{S}} \boldsymbol{u}\right)^{\mathrm{T}} \\
2 \varepsilon^{\mathrm{s}} & =\gamma \boldsymbol{a}_{3}+\boldsymbol{a}_{3} \gamma, \quad 2 \boldsymbol{e}^{\mathrm{s}}=\boldsymbol{\gamma} \\
2 \varepsilon^{\mathrm{b}} & =-\boldsymbol{\nabla}_{\mathcal{S}} \boldsymbol{\theta} \cdot \mathcal{A}-\mathcal{A} \cdot\left(\boldsymbol{\nabla}_{\mathcal{S}} \boldsymbol{\theta}\right)^{\mathrm{T}}-\boldsymbol{\nabla}_{\mathcal{S}} \boldsymbol{u} \cdot \mathcal{B}-\mathcal{B} \cdot\left(\boldsymbol{\nabla}_{\mathcal{S}} \boldsymbol{u}\right)^{\mathrm{T}}
\end{aligned}
$$

The elimination of $\boldsymbol{\gamma}=\nabla_{\mathcal{S}} \boldsymbol{u} \cdot \boldsymbol{a}_{3}-\boldsymbol{\theta}$ changes the shear strain tensor to

$$
2 \varepsilon^{\mathrm{s}}=\left(\nabla_{\mathcal{S}} \boldsymbol{u} \cdot \boldsymbol{a}_{3}-\boldsymbol{\theta}\right) \boldsymbol{a}_{3}+\boldsymbol{a}_{3}\left(\nabla_{\mathcal{S}} \boldsymbol{u} \cdot \boldsymbol{a}_{3}-\boldsymbol{\theta}\right), \quad 2 \boldsymbol{e}^{\mathrm{s}}=\nabla_{\mathcal{S}} \boldsymbol{u} \cdot \boldsymbol{a}_{3}-\boldsymbol{\theta} .
$$

See also [15] for a full derivation (with $\boldsymbol{\theta}$ oriented differently, $\boldsymbol{\theta}_{\text {here }}=-\boldsymbol{\theta}_{\text {there }}$ ). The bilinear form stays as in (6) and (8).

\subsection{The Koiter shell}

While the Naghdi shell is the shell equivalent to the Mindlin-Reissner plate, the Koiter shell is the equivalent to the Kirchhoff plate. The additional hypothesis reads

$$
\boldsymbol{\theta}=\nabla_{\mathcal{S}} \boldsymbol{u} \cdot \boldsymbol{a}_{3}, \quad \boldsymbol{\gamma}=\nabla_{\mathcal{S}} \boldsymbol{u} \cdot \boldsymbol{a}_{3}-\boldsymbol{\theta}=0
$$

for shells. It is a valid assumption only for thin shells.

\subsubsection{Coordinate free Koiter shell formulation}

Direct insertion of the hypothesis into the coordinate free Naghdi shell formulation yields $\boldsymbol{e}^{\mathrm{s}}=0$ and

$$
\begin{aligned}
2 \varepsilon^{\mathrm{m}} & =\nabla_{\mathcal{S}} \boldsymbol{u} \cdot \mathcal{A}+\mathcal{A} \cdot\left(\nabla_{\mathcal{S}} \boldsymbol{u}\right)^{\top} \\
2 \varepsilon^{\mathrm{b}} & =-\nabla_{\mathcal{S}}\left(\nabla_{\mathcal{S}} \boldsymbol{u} \cdot \boldsymbol{a}_{3}\right) \cdot \mathcal{A}-\mathcal{A} \cdot\left(\nabla_{\mathcal{S}}\left(\nabla_{\mathcal{S}} \boldsymbol{u} \cdot \boldsymbol{a}_{3}\right)\right)^{\top}-\nabla_{\mathcal{S}} \boldsymbol{u} \cdot \mathcal{B}-\mathcal{B} \cdot\left(\nabla_{\mathcal{S}} \boldsymbol{u}\right)^{\top} \\
& =-\nabla_{\mathcal{S}} \nabla_{\mathcal{S}} \boldsymbol{u} \cdot \boldsymbol{a}_{3} \cdot \mathcal{A}-\mathcal{A} \cdot\left(\nabla_{\mathcal{S}} \nabla_{\mathcal{S}} \boldsymbol{u} \cdot \boldsymbol{a}_{3}\right)^{\top}
\end{aligned}
$$

with the chain rule $\nabla_{\mathcal{S}}\left(\nabla_{\mathcal{S}} \boldsymbol{u} \cdot \boldsymbol{a}_{3}\right)=\nabla_{\mathcal{S}} \nabla_{\mathcal{S}} \boldsymbol{u} \cdot \boldsymbol{a}_{3}+\nabla_{\mathcal{S}} \boldsymbol{u} \cdot \nabla_{\mathcal{S}} \boldsymbol{a}_{3}=\nabla_{\mathcal{S}} \nabla_{\mathcal{S}} \boldsymbol{u} \cdot \boldsymbol{a}_{3}-\nabla_{\mathcal{S}} \boldsymbol{u} \cdot \boldsymbol{B}$. See also [14] and [15, section 5.2] for a full derivation. 


\subsubsection{Koiter shell in Cartesian coordinates}

Writing the Koiter shell in covariant coordinates like the Naghdi shell leads to formulas which are not so easy to implement for general geometries, see [5, section 4.2.3]. A conforming discretisation of this formulation requires $C^{0}$ elements for both tangential coordinates and $C^{1}$ elements for the normal coordinate.

Alternatively, one can use Cartesian coordinates for the unknowns, see [2]. The coordinate free formulation from above can be reformulated to

$$
\begin{aligned}
2 \varepsilon^{\mathrm{m}} & =\nabla_{\mathcal{S}} \boldsymbol{u} \cdot \mathcal{A}+\mathcal{A} \cdot\left(\nabla_{\mathcal{S}} \boldsymbol{u}\right)^{\top} \\
& =\boldsymbol{u}_{, j} \cdot \boldsymbol{a}_{i}+\boldsymbol{u}_{, i} \cdot \boldsymbol{a}_{j}, \\
2 \varepsilon^{\mathrm{b}} & =-\nabla_{\mathcal{S}} \nabla_{\mathcal{S}} \boldsymbol{u} \cdot \boldsymbol{a}_{3} \cdot \mathcal{A}-\mathcal{A} \cdot\left(\nabla_{\mathcal{S}} \nabla_{\mathcal{S}} \boldsymbol{u} \cdot \boldsymbol{a}_{3}\right)^{\top} \\
& =2\left(\boldsymbol{u}_{, i j} \cdot \boldsymbol{a}_{3}-\Gamma_{i j}^{k} \boldsymbol{u}_{, k} \cdot \boldsymbol{a}_{3}\right) \boldsymbol{a}^{i} \boldsymbol{a}^{j} .
\end{aligned}
$$

There is no need for further derivative evaluation if the unknowns are given in Cartesian coordinates $\boldsymbol{u}=u_{x} \boldsymbol{e}_{x}+u_{y} \boldsymbol{e}_{y}+u_{z} \boldsymbol{e}_{z}$. The Cartesian basis vectors $\boldsymbol{e}_{x}, \boldsymbol{e}_{y}, \boldsymbol{e}_{z}$ are fixed in space and thus the derivatives carry over directly to the unknown coordinates themselves;

$\boldsymbol{u}_{, i}=\left(\frac{\partial}{\partial \eta^{i}} u_{x}\right) \boldsymbol{e}_{x}+\left(\frac{\partial}{\partial \eta^{i}} u_{y}\right) \boldsymbol{e}_{y}+\left(\frac{\partial}{\partial \eta^{i}} u_{z}\right) \boldsymbol{e}_{z}, \quad \boldsymbol{u}_{, i j}=\left(\frac{\partial^{2}}{\partial \eta^{i} \partial \eta^{j}} u_{x}\right) \boldsymbol{e}_{x}+\left(\frac{\partial^{2}}{\partial \eta^{i} \partial \eta^{j}} u_{y}\right) \boldsymbol{e}_{y}+\left(\frac{\partial^{2}}{\partial \eta^{i} \partial \eta^{j}} u_{z}\right) \boldsymbol{e}_{z}$.

This formulation can then easily be implemented. A conforming discretisation requires $C^{1}$ finite elements for all three coordinates $u_{x}, u_{y}, u_{z}$.

\subsection{The principle of Endo-Kimura applied to shells}

The basic idea of Endo and Kimura can also be applied to the Naghdi shell formulation. The total deformation $\boldsymbol{u}$ is split into a bending and a shear part with $\boldsymbol{u}=\boldsymbol{u}_{\mathrm{b}}+\boldsymbol{u}_{\mathrm{s}}$. We start from the coordinate free Naghdi formulation of (9) and (10)

$$
\begin{aligned}
2 \varepsilon^{\mathrm{m}} & =\nabla_{\mathcal{S}} \boldsymbol{u} \cdot \mathcal{A}+\mathcal{A} \cdot\left(\nabla_{\mathcal{S}} \boldsymbol{u}\right)^{\top} \\
2 \boldsymbol{e}^{\mathrm{s}} & =\nabla_{\mathcal{S}} \boldsymbol{u} \cdot \boldsymbol{a}_{3}-\boldsymbol{\theta} \\
2 \varepsilon^{\mathrm{b}} & =-\boldsymbol{\nabla}_{\mathcal{S}} \boldsymbol{\theta} \cdot \mathcal{A}-\mathcal{A} \cdot\left(\boldsymbol{\nabla}_{\mathcal{S}} \boldsymbol{\theta}\right)^{\mathrm{T}}-\boldsymbol{\nabla}_{\mathcal{S}} \boldsymbol{u} \cdot \mathcal{B}-\mathcal{B} \cdot\left(\boldsymbol{\nabla}_{\mathcal{S}} \boldsymbol{u}\right)^{\top}
\end{aligned}
$$

and insert the assumption $\boldsymbol{\theta}=\nabla_{\mathcal{S}} \boldsymbol{u}_{\mathrm{b}} \cdot \boldsymbol{a}_{3}$ to obtain

$$
\begin{aligned}
2 \varepsilon^{\mathrm{m}} & =\nabla_{\mathcal{S}} \boldsymbol{u} \cdot \mathcal{A}+\mathcal{A} \cdot\left(\nabla_{\mathcal{S}} \boldsymbol{u}\right)^{\top} \\
2 \boldsymbol{e}^{\mathrm{s}} & =\nabla_{\mathcal{S}} \boldsymbol{u} \cdot \boldsymbol{a}_{3}-\nabla_{\mathcal{S}} \boldsymbol{u}_{\mathrm{b}} \cdot \boldsymbol{a}_{3}, \\
2 \varepsilon^{\mathrm{b}} & =-\boldsymbol{\nabla}_{\mathcal{S}}\left(\boldsymbol{\nabla}_{\mathcal{S}} \boldsymbol{u}_{\mathrm{b}} \cdot \boldsymbol{a}_{3}\right) \cdot \mathcal{A}-\mathcal{A} \cdot\left(\nabla_{\mathcal{S}}\left(\nabla_{\mathcal{S}} \boldsymbol{u}_{\mathrm{b}} \cdot \boldsymbol{a}_{3}\right)\right)^{\top}-\boldsymbol{\nabla}_{\mathcal{S}} \boldsymbol{u} \cdot \mathcal{B}-\mathcal{B} \cdot\left(\nabla_{\mathcal{S}} \boldsymbol{u}\right)^{\top}
\end{aligned}
$$


as the shell equivalent to the standard Endo-Kimura plate formulation. Hierarchic and decoupled versions can again be obtained by using the unknowns $\boldsymbol{u}$ and $\boldsymbol{u}_{\mathrm{s}}$ with elimination of $\boldsymbol{u}_{\mathrm{b}}$ for the hierarchic version or by using $\boldsymbol{u}_{\mathrm{b}}$ and $\boldsymbol{u}_{\mathrm{s}}$ with elimination of $\boldsymbol{u}$ for the decoupled version. Just like in the special case of a plate the additional gradient condition leads to a slightly stiffer solution than that of the Naghdi shell.

The decoupled version reads

$$
\begin{aligned}
2 \varepsilon^{\mathrm{m}}= & \nabla_{\mathcal{S}}\left(\boldsymbol{u}_{\mathrm{b}}+\boldsymbol{u}_{\mathrm{s}}\right) \cdot \mathcal{A}+\mathcal{A} \cdot\left(\nabla_{\mathcal{S}}\left(\boldsymbol{u}_{\mathrm{b}}+\boldsymbol{u}_{\mathrm{s}}\right)\right)^{\top} \\
2 \boldsymbol{e}^{\mathrm{s}}= & \nabla_{\mathcal{S}} \boldsymbol{u}_{\mathrm{s}} \cdot \boldsymbol{a}_{3}, \\
2 \varepsilon^{\mathrm{b}}= & -\boldsymbol{\nabla}_{\mathcal{S}}\left(\boldsymbol{\nabla}_{\mathcal{S}} \boldsymbol{u}_{\mathrm{b}} \cdot \boldsymbol{a}_{3}\right) \cdot \mathcal{A}-\mathcal{A} \cdot\left(\nabla_{\mathcal{S}}\left(\nabla_{\mathcal{S}} \boldsymbol{u}_{\mathrm{b}} \cdot \boldsymbol{a}_{3}\right)\right)^{\top} \\
& \left.\left.-\nabla_{\mathcal{S}}\left(\boldsymbol{u}_{\mathrm{b}}+\boldsymbol{u}_{\mathrm{s}}\right)\right) \cdot \mathcal{B}-\mathcal{B} \cdot\left(\nabla_{\mathcal{S}}\left(\boldsymbol{u}_{\mathrm{b}}+\boldsymbol{u}_{\mathrm{s}}\right)\right)\right)^{\top}
\end{aligned}
$$

and the product rule $\nabla_{\mathcal{S}}\left(\nabla_{\mathcal{S}} \boldsymbol{u}_{\mathrm{b}} \cdot \boldsymbol{a}_{3}\right)=\nabla_{\mathcal{S}} \nabla_{\mathcal{S}} \boldsymbol{u}_{\mathrm{b}}+\nabla_{\mathcal{S}} \boldsymbol{u}_{\mathrm{b}} \mathcal{B}$ together with $\mathcal{B} \cdot \mathcal{A}=\mathcal{B}$ yields the simplified bending strain

$$
2 \varepsilon^{\mathrm{b}}=-\left(\nabla_{\mathcal{S}} \nabla_{\mathcal{S}} \boldsymbol{u}_{\mathrm{b}} \cdot \boldsymbol{a}_{3}\right) \cdot \mathcal{A}-\mathcal{A} \cdot\left(\nabla_{\mathcal{S}} \nabla_{\mathcal{S}} \boldsymbol{u}_{\mathrm{b}} \cdot \boldsymbol{a}_{3}\right)^{\top}-\nabla_{\mathcal{S}} \boldsymbol{u}_{\mathrm{s}} \cdot \mathcal{B}-\mathcal{B} \cdot\left(\nabla_{\mathcal{S}} \boldsymbol{u}_{\mathrm{s}}\right)^{\top} .
$$

The bending strain features both $\boldsymbol{u}_{\mathrm{b}}$ and $\boldsymbol{u}_{\mathrm{s}}$. Therefore, this formulation is actually not totally decoupled like in the special case of a plate. We still keep the name "decoupled" to link it with the corresponding plate formulation.

We have not yet specified if $\boldsymbol{u}_{\mathrm{s}}$ is from $\mathbb{R}^{3}$ or a smaller subspace. From now on we use $\boldsymbol{u}_{\mathrm{s}} \in \operatorname{span}\left\{\boldsymbol{a}_{3}\right\}$ because it is the equivalent of the plate deflection $w_{\mathrm{s}}$. In contrast, $\boldsymbol{u}_{\mathrm{b}}$ is from the whole $\mathbb{R}^{3}$ because it emulates not only the deflection but also the membrane deformations. It is sensible to use the normal coordinate $\boldsymbol{u}_{\mathrm{s}}=w_{\mathrm{s}} \boldsymbol{a}_{3}$ as an unknown with this assumption. The coordinates of $\boldsymbol{u}_{\mathrm{b}}$ on the other hand are best expressed in Cartesian coordinates via $\boldsymbol{u}_{\mathrm{b}}=u_{\mathrm{b} x} \boldsymbol{e}_{x}+u_{\mathrm{b} y} \boldsymbol{e}_{y}+u_{\mathrm{b} z} \boldsymbol{e}_{z}$. This yields the covariant coordinates

$$
\begin{aligned}
2 \varepsilon_{i j}^{\mathrm{m}} & =\boldsymbol{u}_{\mathrm{b}, i} \cdot \boldsymbol{a}_{j}+\boldsymbol{u}_{\mathrm{b}, j} \cdot \boldsymbol{a}_{i}-2 w_{\mathrm{s}} b_{i j}, \\
2 e_{i}^{\mathrm{s}} & =w_{\mathrm{s}, i}, \\
2 \varepsilon_{i j}^{\mathrm{b}} & =-2 \boldsymbol{u}_{\mathrm{b}, i j} \cdot \boldsymbol{a}_{3}+2 \Sigma_{k} \Gamma_{i j}^{k} \boldsymbol{u}_{\mathrm{b}, k} \cdot \boldsymbol{a}_{3}+2 w_{\mathrm{s}} c_{i j}
\end{aligned}
$$

which can be used for implementing the decoupled Endo-Kimura shell with $C^{1}$ elements for the three Cartesian coordinates of $\boldsymbol{u}_{\mathrm{b}}$ and $C^{0}$ elements for the normal coordinate $w_{\mathrm{s}}$ of $\boldsymbol{u}_{\mathrm{s}}$.

\subsection{Hierarchic Naghdi shell formulation based on Endo-Kimura}

A relaxation of the decoupled Endo-Kimura shell formulation to obtain again the Naghdi shell solution can be done analogously to the relaxation of the according plate model 


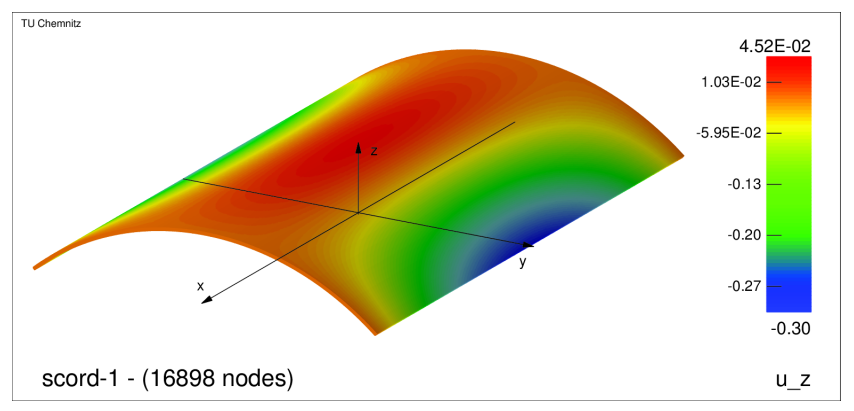

Figure 5: Scordelis-Lo roof with vertical deflection $u_{z}^{3 \mathrm{D}}$

in 2.10. Adding the relaxation angle $\boldsymbol{\rho}=\rho_{1} \boldsymbol{a}^{1}+\rho_{2} \boldsymbol{a}^{2}$ with $\boldsymbol{\theta}=\nabla_{\mathcal{S}} \boldsymbol{u}_{\mathrm{b}} \cdot \boldsymbol{a}_{3}-\boldsymbol{\rho}$ and $\boldsymbol{\gamma}=\nabla_{\mathcal{S}} \boldsymbol{u}_{\mathrm{s}} \cdot \boldsymbol{a}_{3}+\boldsymbol{\rho}$ results in the shear and bending strains

$$
\begin{aligned}
2 \boldsymbol{e}^{\mathrm{s}}= & \nabla_{\mathcal{S}} \boldsymbol{u}_{\mathrm{s}} \cdot \boldsymbol{a}_{3}+\boldsymbol{\rho} \\
2 \varepsilon^{\mathrm{b}}= & -\left(\nabla_{\mathcal{S}} \boldsymbol{\nabla}_{\mathcal{S}} \boldsymbol{u}_{\mathrm{b}} \cdot \boldsymbol{a}_{3}\right) \cdot \mathcal{A}-\mathcal{A} \cdot\left(\nabla_{\mathcal{S}} \boldsymbol{\nabla}_{\mathcal{S}} \boldsymbol{u}_{\mathrm{b}} \cdot \boldsymbol{a}_{3}\right)^{\top}-\nabla_{\mathcal{S}} \boldsymbol{u}_{\mathrm{s}} \cdot \mathcal{B}-\mathcal{B} \cdot\left(\nabla_{\mathcal{S}} \boldsymbol{u}_{\mathrm{s}}\right)^{\top} \\
& +\boldsymbol{\nabla}_{\mathcal{S}} \boldsymbol{\rho} \cdot \mathcal{A}+\mathcal{A} \cdot\left(\nabla_{\mathcal{S}} \boldsymbol{\rho}\right)^{\top}
\end{aligned}
$$

with the covariant coordinates

$$
\begin{aligned}
2 e_{i}^{\mathrm{s}} & =w_{\mathrm{s}, i}+\rho_{i}, \\
2 \varepsilon_{i j}^{\mathrm{b}} & =-2 \boldsymbol{u}_{\mathrm{b}, i j} \cdot \boldsymbol{a}_{3}+2 \Sigma_{k} \Gamma_{i j}^{k} \boldsymbol{u}_{\mathrm{b}, k} \cdot \boldsymbol{a}_{3}+2 w_{\mathrm{s}} c_{i j}+\rho_{i, j}+\rho_{j, i}-2 \Sigma_{k} \Gamma_{i j}^{k} \rho_{k} .
\end{aligned}
$$

The membrane strains are the same as above. A conforming discretisation may be achieved with $C^{1}$ elements for the three Cartesian coordinates of $\boldsymbol{u}_{\mathrm{b}}$ and $C^{0}$ elements for the normal coordinate $w_{\mathrm{s}}$ of $\boldsymbol{u}_{\mathrm{s}}$ and the two covariant coordinates of $\boldsymbol{\rho}$.

\subsection{Numerical example: Scordelis-Lo roof}

The Scordelis-Lo roof was used to validate the exactness of the models and the implementation. The problem with the vertical deflection $u_{z}^{3 \mathrm{D}}$ for is illustrated in Figure 5. A roof section cut out from a right circular cylinder with an opening angle of $80^{\circ}$ rests on a rigid diaphragm with its curved edges. The normal and tangential deformation with respect to the spanning circle are zero at the curved edges (together with their first derivatives in tangential direction); the straight edges are free. The shell thickness is 0.25 , the cylinder radius 25 and the length 50. Isotropic material with $E=4.32 \cdot 10^{8}$ and $\nu=0$ is used and a vertical load per unit surface of -90 is applied. The absolute value of the maximum vertical deflection of $\max \left|u_{z}^{3 \mathrm{D}}\right|=0.3024$, which occurs at the middle of the free edges, is suggested to be used as a benchmark in [10]. Our own overkill solution with 137962 adaptively refined biquadratic Lagrangian elements obtained $\max \left|u_{z}^{3 \mathrm{D}}\right|=0.3043$. We did not make use of symmetries and simulated the whole domain. 


\begin{tabular}{r|rrrr|rrrr}
\hline & \multicolumn{2}{|c|}{ Naghdi bilin. } & \multicolumn{2}{c}{ Naghdi biquadr. } & \multicolumn{2}{c}{ EK shell } & \multicolumn{2}{c}{ hier. Naghdi/EK } \\
\#E1 & $\max \left|u_{z}^{3 \mathrm{D}}\right|$ & \#it & $\max \left|u_{z}^{3 \mathrm{D}}\right|$ & \#it & $\max \left|u_{z}^{3 \mathrm{D}}\right|$ & $\#$ it & $\max \left|u_{z}^{3 \mathrm{D}}\right|$ & \#it \\
\hline 4 & $2.02 \mathrm{E}-3$ & 9 & $3.74 \mathrm{E}-2$ & 36 & 0.2138 & 77 & 0.2809 & 29 \\
16 & $7.72 \mathrm{E}-3$ & 34 & 0.1472 & 267 & 0.2959 & 643 & 0.2960 & 653 \\
64 & $1.99 \mathrm{E}-2$ & 108 & 0.2829 & 661 & 0.3025 & 1011 & 0.3025 & 1047 \\
256 & $3.76 \mathrm{E}-2$ & 255 & 0.3007 & 545 & 0.3030 & 1065 & 0.3031 & 744 \\
1024 & $7.27 \mathrm{E}-2$ & 587 & 0.3026 & 423 & 0.3030 & 605 & 0.3032 & 978 \\
4096 & 0.1485 & 1184 & 0.3038 & 452 & 0.3030 & 709 & 0.3034 & 1629 \\
16384 & 0.2362 & 1764 & 0.3043 & 534 & 0.3030 & 670 & 0.3036 & 2788 \\
\hline
\end{tabular}

Table 10: Results and iteration numbers for Scordelis-Lo roof

\begin{tabular}{r|rrrrrr}
\hline & \multicolumn{2}{|c}{ Naghdi biquadr. } & \multicolumn{2}{c}{ EK shell } & \multicolumn{2}{c}{ hier. Naghdi/EK } \\
\#E1 & $\max \left|u_{z}^{3 \mathrm{D}}\right|$ & \#it & $\max \left|u_{z}^{3 \mathrm{D}}\right|$ & $\#$ it & $\max \left|u_{z}^{3 \mathrm{D}}\right|$ & \#it \\
\hline 4 & $3.77 \mathrm{E}-3$ & 36 & 0.1707 & 59 & 0.2983 & 30 \\
16 & $2.16 \mathrm{E}-2$ & 271 & 0.2181 & 1317 & 0.2184 & 1347 \\
64 & 0.1107 & 1310 & 0.3067 & 5382 & 0.3067 & 6071 \\
256 & 0.2404 & 2949 & 0.8183 & 9494 & 0.3183 & 9655 \\
1024 & 0.3020 & 2712 & 0.3204 & 5615 & 0.3205 & 5636 \\
4096 & 0.3022 & 501 & 0.3206 & 3019 & 0.3206 & 3050 \\
16384 & 0.3197 & 3426 & 0.3206 & 4545 & 0.3206 & 5272 \\
\hline
\end{tabular}

Table 11: Results and iteration numbers for thinner Scordelis-Lo roof

Results obtained with uniform refinement of bilinear as well as biquadratic Lagrangian elements for the standard Naghdi formulation and a combination of bilinear Lagrangian and Bogner-Fox-Schmit elements for the $C^{0}$ and $C^{1}$ parts of the Endo-Kimura shell formulation as well as the hierarchic Naghdi shell formulation based on Endo-Kimura are collected in Table 10. Iteration numbers of all methods are much higher than for their counterparts in the plate example. The iteration numbers of the Koiter shell in cartesian coordinates with BFS elements which are not shown in the table are about the same as those of the Endo-Kimura shell. The bilinear elements show slow $h$-convergence but the $h$-convergence of the other methods appears satisfying.

In order to conduct a locking study we also considered a thinner Scordelis-Lo roof with thickness 0.025 and load -0.9. Results are collected in Table 11. We observe that both Endo-Kimura shell formulations have the best $h$-convergence but at extreme iteration numbers. A better preconditioner tailored for these formulations which might reduce these iteration numbers would be desirable. The Koiter shell in cartesian unknowns with BFS elements shows iteration numbers in the same order of magnitude. 


\section{Conclusion}

We have compared several known and two new plate formulations with respect to their locking behaviour and their numerical efficiency combined with a conforming discretisation. The two new formulations for the Endo-Kimura variant and the original MindlinReissner plate turned out to be locking free and among the most efficient methods for both thick and thin plates.

Extensions of both formulations to shells have been presented. The example problem has shown that the solution of shell problems is more challenging and a better preconditioner might be needed. Nevertheless both methods are locking free and thus suitable for thick and thin shells.

\section{References}

[1] M. Bernadou and K. Hassan. Basis functions for general Hsieh-Clough-Tocher triangles, complete or reduced. International Journal for Numerical Methods in Engineering, 17(5):784-789, 1981.

[2] A. Blouza and H. Le Dret. Existence and uniqueness for the linear Koiter model for shells with little regularity. Quarterly of Applied Mathematics, 57(2):317-337, 1999.

[3] F. K. Bogner, R. L. Fox, and L. A. Schmit. The generation of interelement compatible stiffness and mass matrices by the use of interpolation formulas. In Proceedings of the Conference on Matrix Methods in Structural Mechanics, pages 397-444, 1965.

[4] C. Carstensen and J. Hu. A posteriori error analysis for conforming MITC elements for reissner-mindlin plates. Mathematics of Computation, 77(262):611-632, 2008.

[5] D. Chapelle and K. Bathe. The Finite Element Analysis of Shells: Fundamentals. Computational Fluid and Solid Mechanics. Springer-Verlag GmbH, 2003.

[6] M. Cho, J. Choi, and H.-Y. Roh. Integration of shell FEA with geometric modeling on NURBS surface representation for practical applications. Computer Modeling in Engineering \& Science, 33(1):17-47, 2008.

[7] P. G. Ciarlet. Interpolation error estimates for the reduced Hsieh-Clough-Tocher triangle. Mathematics of Computation, 32(142):335-344, 1978. 
[8] R. Echter. Isogeometric analysis of shells. Dissertation, Bericht Nr. 59, Institut für Baustatik und Baudynamik der Universität Stuttgart, 2013.

[9] M. Endo and N. Kimura. An alternative formulation of the boundary value problem for the Timoshenko beam and Mindlin plate. Journal of Sound and Vibration, 301(1):355 - 373, 2007.

[10] R. H. Macneal and R. L. Harder. A proposed standard set of problems to test finite element accuracy. Finite Elements in Analysis and Design, 1(1):3 - 20, 1985.

[11] A. Meyer. Hierarchical preconditioners for higher order elements and applications in computational mechanics. Preprint Series of the SFB 393 SFB393/99-02, Technische Universität Chemnitz, 1999.

[12] A. Meyer. Programmer's manual for adaptive finite element code SPC-PM 2Ad. Preprint Series of the SFB 393 SFB393/01-18, Technische Universität Chemnitz, 2001.

[13] A. Meyer. Hierarchical preconditioners and adaptivity for Kirchhoff-plates. Chemnitz Scientific Computing Preprints CSC/08-03, Technische Universität Chemnitz, 2008.

[14] A. Meyer. The Koiter shell equation in a coordinate free description - extended. Chemnitz Scientific Computing Preprints CSC/13-01, Technische Universität Chemnitz, 2013.

[15] A. Meyer. The linear Naghdi shell equation in a coordinate free description. Chemnitz Scientific Computing Preprints CSC/13-03, Technische Universität Chemnitz, 2013.

[16] B. Oesterle, E. Ramm, and M. Bischoff. A shear deformable, rotation-free isogeometric shell formulation. Computer Methods in Applied Mechanics and Engineering, 307(Supplement C):235 - 255, 2016.

[17] M. Weise. Simplified calculation of rHCT basis functions for an arbitrary splitting. Chemnitz Scientific Computing Preprints CSC/15-01, Technische Universität Chemnitz, 2015.

[18] M. Weise. Adaptive FEM for NURBS surface shells. PAMM, 16(1):773-774, 2016. 
Some titles in this CSC preprint series:

10-01 A. Meyer, P. Steinhorst. Modellierung und Numerik wachsender Risse bei piezoelektrischem Material. May 2010.

10-02 M. Balg, A. Meyer. Numerische Simulation nahezu inkompressibler Materialien unter Verwendung von adaptiver, gemischter FEM. Juni 2010.

10-03 M. Weise, A. Meyer. Grundgleichungen für transversal isotropes Materialverhalten. Juli 2010.

10-04 M. K. Bernauer, R. Herzog. Optimal Control of the Classical Two-Phase Stefan Problem in Level Set Formulation. October 2010.

11-01 P. Benner, M.-S. Hossain, T. Stykel. Low-rank iterative methods of periodic projected Lyapunov equations and their application in model reduction of periodic descriptor systems. February 2011.

11-02 G. Of, G. J. Rodin, O. Steinbach, M. Taus. Coupling Methods for Interior Penalty Discontinuous Galerkin Finite Element Methods and Boundary Element Methods. September 2011.

12-01 J. Rückert, A. Meyer. Kirchhoff Plates and Large Deformation. April 2012.

12-02 A. Meyer. The Koiter shell equation in a coordinate free description. February 2012.

12-03 M. Balg, A. Meyer. Fast simulation of (nearly) incompressible nonlinear elastic material at large strain via adaptive mixed FEM. July 2012.

13-01 A. Meyer. The Koiter shell equation in a coordinate free description - extended. September 2013 .

13-02 R. Schneider. With a new refinement paradigm towards anisotropic adaptive FEM on triangular meshes. September 2013.

13-01 A. Meyer. The linear Naghdi shell equation in a coordinate free description. November 2013.

14-01 A. Meyer. Programmbeschreibung SPC-PM3-AdH-XX - Teil 1. März 2014.

14-02 A. Meyer. Programmbeschreibung SPC-PM3-AdH-XX - Teil 2. April 2014.

14-03 J. Glänzel, R. Unger. High Quality FEM-Postprocessing and Visualization Using a Gnuplot Based Toolchain. July 2014. 
14-04 M. Weise. A note on the second derivatives of rHCT basis functions. October 2014.

15-01 M. Weise. Simplified calculation of rHCT basis functions for an arbitrary splitting. January 2015.

15-02 M. Weise. A note on the second derivatives of rHCT basis functions - extended. January 2015.

15-03 R. Springer, A. Meyer. Basics of Linear Thermoelasticity. January 2015.

15-04 R. Schneider, G. Wachsmuth. A-posteriori error estimation for control-constrained, linear-quadratic optimal control problems. May 2015.

15-05 N. Lang, J. Saak, T. Stykel. Balanced truncation model reduction for linear time-varying systems. October 2015.

The complete list of CSC and SFB393 preprints is available via

http://www.tu-chemnitz.de/mathematik/csc/ 

Chemnitz Scientific Computing Preprints - ISSN 1864-0087 